Алгебра и анализ

Том. 17 (2005), вып. 5
St. Petersburg Math. J.

Vol. 17 (2006), No. 5, Pages 835-864

S 1061-0022(06)00932-0

Article electronically published on July 27, 2006

\title{
ON A MATHEMATICAL MODEL OF IRREVERSIBLE QUANTUM GRAPHS
}

\author{
M. Z. SOLOMYAK
}

In fond memory of Ol'ga Aleksandrovna Ladyzhenskaya

\begin{abstract}
The "irreversible quantum graph" model, suggested by U. Smilansky, is considered. Mathematically, the problem is in the investigation of the spectrum of the operator $\mathbf{A}_{\alpha}$ determined by an infinite system of ordinary differential equations on a graph and by a system of boundary conditions, such as conditions on the jumps of derivatives. The operator depends on a parameter $\alpha \geq 0$ involved in the boundary conditions only.

In the paper, the point spectrum and the absolute continuous spectrum of the operator $\mathbf{A}_{\alpha}$ are studied in detail in their dependence on $\alpha$. Some special effects appear, the main one being a "phase transition" for some value $\alpha=\alpha_{0}$ that depends on the geometry of the graph: the spectral properties of the operator for $\alpha<\alpha_{0}$ and $\alpha>\alpha_{0}$ differ greatly.
\end{abstract}

\section{$\S 0$. INTRODUCTION}

1. Quantum graphs. In recent years, the term "quantum graph" has become commonplace in the theory of differential equations on graphs. A quantum graph is a system consisting of a metric graph $\Gamma$ and a selfadjoint operator acting in $L^{2}(\Gamma)$. Usually, the role of the operator is played by the Laplacian. The theory of quantum graphs is a very popular part of the general spectral theory of differential operators; see the survey [9] and the wide list of references therein, and also the special issue of the journal Waves in Random Media (14 (2004), no. 1).

2. Informal setting of the problem. In the paper [13, published in the abovementioned issue, Smilansky suggested a mathematical model called the "irreversible quantum graph". In that model, an interaction is studied between a quantum graph and an attached system of one-dimensional harmonic oscillators. More precisely, along with the dynamical system generated by the Laplacian in $L^{2}(\Gamma)$, we consider the dynamical system in $L^{2}\left(\mathbb{R}^{K}\right)$ generated by the Hamiltonian $H_{\mathrm{osc}}=\sum_{k=1}^{K} h_{k}$ with

$$
h_{k}=\frac{1}{2}\left(-\frac{\partial^{2}}{\partial q_{k}{ }^{2}}+\omega_{k} q_{k}^{2}\right), \quad \omega_{k}>0, \quad k=1, \ldots, K .
$$

In what follows, the points on the graph are denoted by $x$ and the points in $L^{2}\left(\mathbb{R}^{K}\right)$ by $\mathbf{q}=\left(q_{1}, \ldots, q_{K}\right)$.

Consider the operator

$$
\mathbf{A}_{0}=-\Delta \otimes I+I \otimes H_{\mathrm{osc}}
$$

2000 Mathematics Subject Classification. Primary 35Q40, 34L40.

Key words and phrases. Quantum graphs, spectrum, Jacobi matrices. 
in $L^{2}\left(\Gamma \times \mathbb{R}^{K}\right)$. This operator is given by the differential expression

$$
\mathcal{A} U=-\Delta_{x} U+\frac{1}{2} \sum_{k=1}^{K}\left(-\frac{\partial^{2} U}{\partial q_{k}{ }^{2}}+\omega_{k} q_{k}^{2} U\right)
$$

and is selfadjoint on the natural domain. The terms in (0.2) do not interact.

The interaction is introduced via a system of conditions imposed on the derivative $U_{x}^{\prime}$ at $x=o_{k}, k=1, \ldots, K$. Here the $o_{k}$ are some chosen points on the graph. We say that the oscillator $h_{k}$ is attached to the graph $\Gamma$ at the point $o_{k}$.

The conditions imposed on $U_{x}^{\prime}$ are of the form

$$
\left[U_{x}^{\prime}\right]\left(o_{k}, \mathbf{q}\right)=\alpha_{k} q_{k} U\left(o_{k}, \mathbf{q}\right), \quad k=1, \ldots, K .
$$

Here $\left[U_{x}^{\prime}\right](\cdot)$ is the combination of derivatives that appears in Kirchhoff's condition, well known in the theory of functions on graphs. A precise definition of $\left[U_{x}^{\prime}\right](\cdot)$ is given in (1.1). Now, we only note that if $\Gamma=\mathbb{R}$, then $\left[U_{x}^{\prime}\right](o, \mathbf{q})$ coincides with the jump of the derivative in $x$ at the point $(o, \mathbf{q})$. Next, the $\alpha_{k}$ in (0.4) are real-valued parameters; they constitute the $K$-dimensional coupling parameter $\alpha=\left\{\alpha_{1}, \ldots, \alpha_{K}\right\}$. This parameter expresses the strength of the interaction.

By $\mathbf{A}_{\alpha}$ we denote the operator defined by the differential equation (0.3) and the system of conditions (0.4). We are going to describe the spectrum of the dynamical system generated by the Hamiltonian $\mathbf{A}_{\alpha}$. Mathematically, the problem reduces to realizing $\mathbf{A}_{\alpha}$ as a selfadjoint operator in $L^{2}\left(\Gamma \times \mathbb{R}^{K}\right)$ and to describing its spectrum. Note that the change of the sign of $\alpha_{k}$ is equivalent to the substitution $q_{k} \mapsto-q_{k}$, which does not affect the spectrum. Thus, in the sequel we assume that $\alpha_{k} \geq 0, k=1, \ldots, K$.

At first sight, the problem is not difficult. Indeed, the differential equation (0.3) is standard, and conditions (0.4) seem rather simple. The problem is typical for perturbation theory: the spectrum of $\mathbf{A}_{0}$ can be found easily by separation of variables and, at the same time, the perturbation introduced by conditions (0.4) does not appear too strong. Though this perturbation changes the domain of the operator, we can try to study the problem in terms of quadratic forms.

However, this first impression is deceptive. The perturbation turns out to be bounded but not compact relative to the quadratic form of the operator $\mathbf{A}_{0}$. Therefore, the standard perturbation theory methods do not provide sufficient information. In the light of these difficulties, the problem is very interesting and nontrivial from a purely mathematical point of view.

For $K=1$, a fairly complete study of the problem has turned out to be possible, despite the failure of the requirement that the perturbation be relatively compact. Our analysis is based on combining the methods of the extension theory for symmetric operators, the variational techniques, the theory of analytic operator-valued functions, and, especially, the theory of operators defined in $\ell^{2}$ by Jacobi matrices. The Jacobi matrices arise naturally in the analysis of the problem because of quite a special way in which the coordinate $q=q_{1}$ is involved in condition (0.4). Apart from their crucial technical role in the investigation, Jacobi matrices also appear in the formulation of the main results.

3. Main effects. The family of operators $\mathbf{A}_{\alpha}$ has a series of unusual properties. Here, we describe these properties in the case where $\Gamma=\Gamma_{d}$ is a graph generated by $d$ edges of infinite length with common vertex $o$. In this case, the spectrum of the operator $\mathbf{A}_{0}$ is absolutely continuous and fills the half-line $[1 / 2, \infty)$. It is not difficult to write an expression for the corresponding multiplicity function, but we shall not do this here. If $0<\alpha \sqrt{2}<d$, then the absolutely continuous spectrum of $\mathbf{A}_{\alpha}$ and its multiplicity function coincide with those for the operator $\mathbf{A}_{0}$. Also, eigenvalues arise in the interval $(0,1 / 2)$. The number of eigenvalues grows like $O\left((d-\alpha \sqrt{2})^{-1 / 2}\right)$ as $\alpha \sqrt{2} \nearrow d$. For 
$\alpha \sqrt{2}=d$, these eigenvalues disappear, and a new branch of absolutely continuous spectrum arises and fills the half-line $\lambda \geq 0$. For $\alpha \sqrt{2}>d$ the absolutely continuous spectrum fills the entire real axis, and the point spectrum is empty. In the general case the picture is more complicated, but the main features stay the same. Complete formulations are presented at the end of $\S 3$. They show that for an arbitrary graph $\Gamma$ a "phase transition" occurs for some value $\alpha=\alpha_{0}$ of the parameter. The structure of the spectrum of $\mathbf{A}_{\alpha}$ is drastically different for $\alpha<\alpha_{0}$ and $\alpha>\alpha_{0}$. Precisely this effect, discovered for the first time by Smilansky in 13] (in different terms and without strict mathematical proof) was interpreted as the irreversibility of a quantum system.

Our main goal in this paper is to present the results obtained to date and to attract the reader's attention in the subject. The results for $K>1$ are discussed in $\S 12$. In other sections we assume that $K=1$. Thus, all quantities in the differential equation (0.3) and condition (0.4) are denoted by $q, o$ and $\alpha$ without indices. Next, in (0.3) we assume that $\omega=\omega_{1}=1$; this can be achieved by rescaling.

The author's interest in the subject was motivated by his contacts with U. Smilansky. The first rigorous results on the properties of the operators $\mathbf{A}_{\alpha}$ were obtained in the paper [14, which was published simultaneously with [13. The present paper is based on the results of $[14,15,12]$. Note that only star graphs were treated in those papers. Unlike 14, 15, 12, here we consider a fairly general situation: it is assumed that the graph becomes compact after removing finitely many edges of infinite length. We show that only a minimal complication of the technique is needed to cover this general case.

4. Notation. Here we introduce some notation used in the paper. For a selfadjoint operator $\mathbf{T}$ in the Hilbert space $\mathcal{H}$, by $E^{\mathbf{T}}$ and $\sigma(\mathbf{T})$ we denote its spectral measure and its spectrum, respectively. We write $\sigma_{\text {a.c. }}(\mathbf{T})$ and $\sigma_{p}(\mathbf{T})$ for the absolutely continuous spectrum and the point spectrum of the operator. Next, the multiplicity function for the absolutely continuous spectrum is denoted by $\mathfrak{m}_{\text {a.c. }}(\lambda ; \mathbf{T})$ (see [1] and [16]). The multiplicity function is defined up to Lebesgue measure equivalence.

For any real number $s$, we denote

$$
N_{+}(s ; \mathbf{T})=\operatorname{dim} E^{\mathbf{T}}(s, \infty) \mathcal{H}, \quad N_{-}(s ; \mathbf{T})=\operatorname{dim} E^{\mathbf{T}}(-\infty, s) \mathcal{H} .
$$

The inequality $N_{ \pm}(s ; \mathbf{T})<\infty$ is equivalent to the fact that the spectrum of the operator $\mathbf{T}$ on the half-line $\pm \lambda \in(s, \infty)$ consists of finitely many eigenvalues (with regard to multiplicity); the quantities $N_{ \pm}(s ; \mathbf{T})$ are equal to the number of such eigenvalues.

The Schatten classes of compact operators (see [4]) are denoted by $\mathfrak{S}_{p}$. In particular, $\mathfrak{S}_{2}$ is the Hilbert-Schmidt class and $\mathfrak{S}_{1}$ is the trace class.

Let $\left\{a_{n}\right\}, n \geq 0$, and $\left\{b_{n}\right\}, n \geq 1$, be two number sequences. We use the notation $\mathbf{J}\left(\left\{a_{n}\right\},\left\{b_{n}\right\}\right)$ for the Jacobi matrix

$$
\mathbf{J}\left(\left\{a_{n}\right\},\left\{b_{n}\right\}\right)=\left(\begin{array}{ccccc}
a_{0} & b_{1} & 0 & 0 & \ldots \\
b_{1} & a_{1} & b_{2} & 0 & \ldots \\
0 & b_{2} & a_{2} & b_{3} & \ldots \\
\ldots & \ldots & \ldots & \ldots & \ldots
\end{array}\right) .
$$

We do not distinguish between the matrix $\mathbf{J}\left(\left\{a_{n}\right\},\left\{b_{n}\right\}\right)$ and the operator defined by this matrix in $\ell^{2}\left(\mathbb{N}_{0}\right)$. Here, $\mathbb{N}_{0}=\{0,1, \ldots\}$.

5. Acknowledgments. I am grateful to U. Smilansky for attracting my attention to this problem and for numerous discussions. My thanks are due to S. N. Naboko for collaboration in the joint paper [12]. The technique developed in [12] applies almost without changes to the more general situation discussed in the present paper. Finally, I would like to thank W. D. Evans, P. Kuchment, B. S. Pavlov, and D. R. Yafaev for useful discussions. 


\section{$\S 1$. THE LAPLACIAN ON A GRAPH}

1. Metric graphs. A metric graph is a graph whose edges are viewed as segments of the real axis rather than pairs of points (vertices). We describe the class $\mathfrak{G}$ of metric graphs to be dealt with in what follows. The graph $\Gamma$ is assumed to be connected. The set $\mathcal{V}=\mathcal{V}(\Gamma)$ of its vertices and the set $\mathcal{E}=\mathcal{E}(\Gamma)$ of its edges are assumed finite. We admit edges of infinite length (such edges have only one vertex). We say that two vertices are neighbors if they are connected by an edge. For a vertex $v \in \mathcal{V}$, we denote by $d(v)$ its degree, i.e., the number of edges outgoing from it. The boundary $\partial \Gamma$ consists of all edges $v$ for which $d(v)=1$. The boundary may be empty. Any point $x \in \Gamma \backslash \mathcal{V}$ can serve as an additional vertex. Then $d(x)=2$. For simplicity, we consider graphs without loops or multiple edges. This means that no vertex is its own neighbor and that any two neighboring vertices are connected by one edge only. However, this restriction is not essential because we always can add appropriate vertices.

The simplest graphs are the so-called star graphs. A star graph is generated by $d \geq 1$ edges with a common vertex. The graph $\Gamma_{d}$ described in the Introduction is of this type. It has only one vertex $v$ and, obviously, $d(v)=d$.

As usual, the distance $\rho(x, y)$ between two points $x, y \in \Gamma$ is defined as the length of the shortest polygonal path on $\Gamma$ connecting these points. The topology of $\Gamma$ is induced by this metric. The length of an edge $e \in \mathcal{E}$ is denoted by $|e|$. A graph of class $\mathfrak{G}$ is compact if and only if all its edges have finite length. For any vertex $v \in \mathcal{V}$, we denote by $\mathcal{S}(v)$ its star vicinity, i.e., the union of all edges for which $v$ is a vertex.

Below we fix a point $o \in \Gamma$. We can always assume that this point is a vertex. The following characteristics of the graph $\Gamma$ and the pair $(\Gamma, o)$ are of particular interest for us, because they appear in the formulation of the main results: the number $M(\Gamma)$ of edges $e \in \mathcal{E}$ of infinite length, and the degree $d(o)$ of the vertex $o$.

For some auxiliary technical results, we also need the quantity

$$
\varepsilon(\Gamma, o)=\min \{|e|: e \text { is an edge and } e \subset \mathcal{S}(o)\} .
$$

For a function $u$ on a graph, the derivative $u^{\prime}$ along any edge $e$ can be defined naturally, provided that the orientation on $e$ is indicated. For the second-order derivative such indication is not necessary. If $v$ is a vertex, then it is natural to identify every edge $e \subset \mathcal{S}(v)$ with the segment $[0,|e|]$ (or with the half-line $[0, \infty)$ if $|e|=\infty$ ) so that $t=0$ corresponds to the point $v$. This fixes an orientation on the edges $e \subset \mathcal{S}(v)$. We introduce the notation

$$
\left[u^{\prime}\right](v)=\sum_{e \in \mathcal{S}(v)}\left(\left.u\right|_{e}\right)^{\prime}(0)
$$

Sometimes, the same quantity will be denoted by $\left.\left[u_{x}^{\prime}\right]\right|_{x=v}$.

The measure $d x$ on the graph is induced by the Lebesgue measure on the edges, and $L^{2}(\Gamma):=L^{2}(\Gamma, d x)$. By definition, the Sobolev space $H^{1}(\Gamma)$ consists of all continuous functions on $\Gamma$ such that $\left.u\right|_{e} \in H^{1}(e)$ for all edges $e \in \mathcal{E}$ and

$$
\|u\|_{H^{1}(\Gamma)}^{2}:=\int_{\Gamma}\left(\left|u^{\prime}\right|^{2}+|u|^{2}\right) d x<\infty .
$$

The space $H^{1}(\Gamma)$ with this metric is a Hilbert space.

2. The Laplacian on $\Gamma$. The Laplacian $-\Delta$ on the graph $\Gamma$ can be defined as the selfadjoint operator in $L^{2}(\Gamma)$ generated by the quadratic form $\int_{\Gamma}\left|u^{\prime}\right|^{2} d x$ with the domain $H^{1}(\Gamma)$; we emphasize that by the Laplacian we mean the operator $-\Delta$. It is not difficult to describe the domain Dom $\Delta$. Namely, a function $u \in H^{1}(\Gamma)$ belongs to Dom $\Delta$ if and 
only if $u \in H^{2}(e)$ for any edge $e, \sum_{e \subset \mathcal{E}} \int_{e}\left|u^{\prime \prime}\right|^{2} d x<\infty$, and the following Kirchhoff's condition is fulfilled at every vertex:

$$
\left[u^{\prime}\right](v)=0, \quad v \in \mathcal{V} .
$$

This condition is natural in the context of the calculus of variations. Obviously, for $u \in \operatorname{Dom} \Delta$ we have $\Delta u(x)=u^{\prime \prime}(x)$ for $x \notin \mathcal{V}$.

For the vertices $v \in \partial \Gamma$, condition (1.2) reduces to the Neumann condition. Some other boundary conditions on $\partial \Gamma$ (e.g., the Dirichlet condition) are also possible, as well as other conditions at vertices $v \notin \partial \Gamma$. However, to be definite, we shall mainly consider the Laplacian under the conditions (1.2) for all vertices.

It is not difficult to describe the spectral properties of the Laplacian on $\Gamma$. Its spectrum is discrete if and only if the graph $\Gamma$ is compact. Otherwise, the spectrum has an absolutely continuous part which fills the half-line $[0, \infty)$. The multiplicity of the absolutely continuous spectrum is constant and is equal to $M(\Gamma)$. This can be proved easily with the help of the splitting principle. Since the Laplacian is nonnegative, we have $\sigma(-\Delta)=[0, \infty)$ in the noncompact case, i.e., the entire spectrum coincides geometrically with the absolutely continuous spectrum. The operator may have eigenvalues embedded in the absolutely continuous spectrum. In the case of a star graph, such eigenvalues always exist if the graph has at least two edges whose lengths are finite and commensurable. This can be verified by a direct calculation (see [14, §3]).

The Neumann condition on $\partial \Gamma$ implies that the point $\lambda=0$ belongs to the spectrum. In the case of the Dirichlet condition, 0 belongs to $\sigma(-\Delta)$ only if $\Gamma$ is noncompact.

\section{§2. The Selfadjoint operator $\mathbf{A}_{\alpha}$}

1. Precise statement of the problem. Reduction to an infinite system of differential equations on $\Gamma$. In accordance with what was said in the Introduction, we fix a point $o \in \Gamma$, to be regarded as a vertex. We consider a family of operators $\mathbf{A}_{\alpha}$ in $L^{2}(\Gamma \times \mathbb{R})$. Each operator $\mathbf{A}_{\alpha}$ is determined by the differential expression

$$
\mathcal{A} U=-\Delta_{x} U+\frac{1}{2}\left(-\frac{\partial^{2} U}{\partial q^{2}}+q^{2} U\right), \quad x \neq \mathcal{V}
$$

and the conditions

$$
\left[U_{x}^{\prime}\right](v, q)=0, \quad v \neq o ; \quad\left[U_{x}^{\prime}\right](o, q)=\alpha q U(o, q), \quad q \in \mathbb{R},
$$

at the vertices.

Yet, these conditions do not determine the domain on which the operator $\mathbf{A}_{\alpha}$ is selfadjoint. To describe this domain, it is useful to pass to another representation of the operator. It is natural to use an expansion with respect to the Hermite functions $\chi_{n}(q)=e^{-q^{2} / 2} H_{n}(q), n=0,1, \ldots$. Here $H_{n}(q)$ is the Hermite polynomial normalized by the condition $\left\|\chi_{n}\right\|_{L^{2}(\mathbb{R})}=1$. The functions $\chi_{n}(q)$ satisfy the recurrence equation

$$
\sqrt{n+1} \chi_{n+1}(q)-\sqrt{2} q \chi_{n}(q)+\sqrt{n} \chi_{n-1}(q)=0,
$$

which can be deduced easily from the well-known recurrence equation for the Hermite polynomials.

So, let

$$
U(x, q)=\sum_{n \in \mathbb{N}_{0}} u_{n}(x) \chi_{n}(q), \quad \mathbb{N}_{0}:=\{0,1, \ldots\} .
$$

We often identify the function $U(x, q)$ with the sequence $\left\{u_{n}(x)\right\}$, and write $U \sim\left\{u_{n}\right\}$. This identification is a unitary transformation of the space $L^{2}(\Gamma \times \mathbb{R})$ onto the Hilbert 
space $\mathfrak{H}=\ell^{2}\left(\mathbb{N}_{0} ; L^{2}(\Gamma)\right)$. Substituting (2.4) in (2.1) formally, we obtain

$$
\mathcal{A} U \sim\left\{f_{n}\right\}, f_{n}(x)=-u_{n}^{\prime \prime}(x)+(n+1 / 2) u_{n}(x), \quad x \notin \mathcal{V}, \quad n \in \mathbb{N}_{0} .
$$

Conditions $(2.2)$ for the vertices $v \neq o$ reduce to the system of conditions

$$
\left[u_{n}^{\prime}\right](v)=0, \quad v \neq o, n \in \mathbb{N}_{0} .
$$

For the vertex $o$, condition (2.2) yields

$$
\sum_{n \in \mathbb{N}_{0}} \chi_{n}(q)\left[u_{n}^{\prime}\right](o)=\alpha \sum_{n \in \mathbb{N}_{0}} q \chi_{n}(q) u_{n}(o) .
$$

Recalling (2.3), we obtain the conditions

$$
\sqrt{2}\left[u_{n}^{\prime}\right](o)=\alpha\left(\sqrt{n+1} u_{n+1}(o)+\sqrt{n} u_{n-1}(o)\right), \quad n \in \mathbb{N}_{0} .
$$

For $n=0$, only the first term is present on the right-hand side of (2.7).

2. Selfadjoint realization of the operator $\mathbf{A}_{\alpha}$. For any $\alpha \geq 0$, we define a linear subset $\mathcal{D}_{\alpha} \subset \mathfrak{H}$. An element $U \sim\left\{u_{n}\right\} \in \mathfrak{H}$ belongs to $\mathcal{D}_{\alpha}$ if and only if

1) $u_{n} \in H^{1}(\Gamma)$ for any $n \in \mathbb{N}_{0}, u_{n} \in H^{2}(e)$ for any edge $e$, and

$$
\sum_{n \in \mathbb{N}_{0}} \int_{\Gamma}\left|-u_{n}^{\prime \prime}+(n+1 / 2) u_{n}\right|^{2} d x<\infty
$$

2) conditions (2.6) and (2.7) are fulfilled at the vertices.

Theorem 2.1. Let $\mathcal{A}$ be the operator given by formula (2.1), or equivalently, by relations (2.5). For any $\alpha \geq 0$ the operator $\mathbf{A}_{\alpha}:=\mathcal{A}\left\lceil\mathcal{D}_{\alpha}\right.$ is selfadjoint in $\mathfrak{H}$.

The proof will be given in $\S 4$ after some technical preparations.

3. The spectrum of the operator $\mathbf{A}_{0}$. The spectrum $\sigma\left(\mathbf{A}_{0}\right)$ can be investigated easily with the help of separation of variables.

Theorem 2.2. The spectrum $\sigma\left(\mathbf{A}_{0}\right)$ is a subset of the half-line $\lambda \geq 1 / 2$. The spectrum is discrete if and only if the graph $\Gamma$ is compact. Otherwise, $\sigma\left(\mathbf{A}_{0}\right)=\sigma_{\text {a.c. }}\left(\mathbf{A}_{0}\right)=[1 / 2, \infty)$, and

$$
\mathfrak{m}_{\text {a.c. }}\left(\lambda ; \mathbf{A}_{0}\right)=M(\Gamma) n, \quad|\lambda-n|<1 / 2, \quad n \in \mathbb{N} .
$$

The operator $\mathbf{A}_{0}$ may also have embedded eigenvalues.

Proof. The definition of $\mathbf{A}_{0}$ implies that this operator expands into an orthogonal sum, namely,

$$
\mathbf{A}_{0}=\sum_{n \in \mathbb{N}_{0}}^{\oplus}(-\Delta+(n+1 / 2))
$$

where $-\Delta$ is the Laplacian on $\Gamma$. Thus, the spectrum of $\mathbf{A}_{0}$ is the union of the spectra of the operators $-\Delta+(n+1 / 2)$ for all $n \in \mathbb{N}_{0}$. Now, the theorem follows from the description (see Subsection 1.2) of the spectrum of the Laplacian.

\section{§3. Formulation of the main Results}

Here we describe the spectrum $\sigma\left(\mathbf{A}_{\alpha}\right)$, its absolutely continuous part $\sigma_{\text {a.c. }}\left(\mathbf{A}_{\alpha}\right)$, and the point part $\sigma_{p}\left(\mathbf{A}_{\alpha}\right)$ for different $\alpha>0$. Most likely, the singular continuous spectrum is absent, but the techniques used in the paper do not allow us to justify this. Observe nevertheless that the theorems below show at least that the essential spectrum $\sigma_{\text {ess }}\left(\mathbf{A}_{\alpha}\right)$ coincides geometrically with $\sigma_{\text {a.c. }}\left(\mathbf{A}_{\alpha}\right)$.

The first result deals with the case of small $\alpha$. 
Theorem 3.1. For $0<\alpha \sqrt{2}<d(o)$, the following statements are true.

1) The absolutely continuous spectrum of the operator $\mathbf{A}_{\alpha}$ and its multiplicity function are the same as for the operator $\mathbf{A}_{0}$.

2) Below the threshold point $1 / 2$, the spectrum is always nonempty and consists of a finite number $N_{-}\left(1 / 2 ; \mathbf{A}_{\alpha}\right)$ of eigenvalues with finite multiplicity.

3) The number $N_{-}\left(1 / 2 ; \mathbf{A}_{\alpha}\right)$ grows infinitely as $\alpha \sqrt{2} \nearrow d(o)$, and the following asymptotic formula is valid:

$$
N_{-}\left(1 / 2 ; \mathbf{A}_{\alpha}\right) \sim \frac{1}{4 \sqrt{2(\mu(\alpha)-1)}}, \quad \mu(\alpha)=\frac{d(o)}{\alpha \sqrt{2}} .
$$

We recall (see Subsection 1.2) that at the vertices $v \neq o$ condition (2.2) reduces to the Neumann condition. Note that the statement that the spectrum on the half-line $\lambda<1 / 2$ is nonempty may fail if the Neumann condition on $\partial \Gamma$ is replaced by another boundary condition.

In the borderline case where $\alpha \sqrt{2}=d(o)$, the picture changes.

Theorem 3.2. For $\alpha \sqrt{2}=d(o)$, the following statements are true.

1) For any graph $\Gamma \in \mathfrak{G}$, the absolutely continuous spectrum of the operator $\mathbf{A}_{\alpha}$ fills the half-line $\lambda \geq 0$, and for a.e. $\lambda$ we have

$$
\mathfrak{m}_{\text {a.c. }}\left(\lambda ; \mathbf{A}_{\alpha}\right)=\mathfrak{m}_{\text {a.c. }}\left(\lambda ; \mathbf{A}_{0}\right)+1 .
$$

2) The spectrum on the half-line $\lambda<0$ is discrete.

3) The operator has no eigenvalues in the interval $(0,1 / 2)$.

The negative spectrum of the operator $\mathbf{A}_{d(o) / \sqrt{2}}$ can be empty. This is the case, e.g., if $\Gamma=\Gamma_{d}$ and $o$ is the only vertex of the graph.

Theorem 3.3. For $\alpha \sqrt{2}>d(o)$, the following statements are true.

1) $\sigma_{\text {a.c. }}\left(\mathbf{A}_{\alpha}\right)=\mathbb{R}$ and the multiplicity function is given by (3.2) for a.e. $\lambda \in \mathbb{R}$.

2) The eigenvalues may lie on the half-line $\lambda \geq 1 / 2$ only.

Theorems 3.1-3.3 show how the spectrum of $\mathbf{A}_{\alpha}$ changes as $\alpha$ grows. For $\alpha \sqrt{2}<d(o)$ the absolutely continuous spectrum is empty if the graph $\Gamma$ is compact; otherwise the absolutely continuous spectrum coincides with the half-line $\lambda \geq 1 / 2$. Eigenvalues arise on the half-line $\lambda<1 / 2$. The number of eigenvalues grows in accordance with the asymptotic formula (3.1) as $\alpha \sqrt{2} \nearrow d(o)$. For $\alpha \sqrt{2}=d(o)$, the eigenvalues in $(0,1 / 2)$ disappear, but a new branch of the absolutely continuous spectrum appears instead. This branch fills the half-line $\lambda \geq 0$. The operator $\mathbf{A}_{d(o) / \sqrt{2}}$ may also have negative eigenvalues. For $\alpha \sqrt{2}>d(o)$ the absolutely continuous spectrum fills the entire real axis. The point spectrum in $(-\infty, 1 / 2)$ disappears.

For the graphs $\Gamma_{d}$, the picture can be refined (see the discussion in Subsection 0.3). In addition to Theorems 3.1-3.3, the following statement is true.

Proposition 3.4. Let $\Gamma=\Gamma_{d}, d \geq 1$. Then for $\alpha \sqrt{2}<d$ the operator $\mathbf{A}_{\alpha}$ is positive definite and has no eigenvalues on the half-line $\lambda \geq 1 / 2$. If $\alpha \sqrt{2} \geq d$, then $\sigma_{p}\left(\mathbf{A}_{\alpha}\right)=\varnothing$.

It is natural to ask what is the source of the additional branch of the absolutely continuous spectrum that appears for $\alpha \sqrt{2} \geq d(o)$. The answer can be given in terms of the Jacobi matrix

$$
\mathbf{J}^{\circ}(\mu)=\mathbf{J}\left(\{2 \mu(n+1 / 2)\},\left\{q_{n}\right\}\right)
$$

(see (0.5)), where

$$
q_{n}=n^{1 / 2}\left(n^{2}-1 / 4\right)^{1 / 4}
$$


The operator $\mathbf{J}^{\circ}(\mu)$, defined initially on sequences with finitely many nonzero elements, is essentially selfadjoint in $\ell^{2}$. We use the same notation $\mathbf{J}^{\circ}(\mu)$ for its unique selfadjoint extension.

Proposition 3.5. Let $\mathbf{J}^{\circ}(\mu)$ be the Jacobi operator given by (3.3) and (3.4). For $\mu<1$ its spectrum is discrete; for $\mu \geq 1$ the spectrum is absolutely continuous. Moreover,

$$
\begin{aligned}
\sigma_{\text {a.c. }}\left(\mathbf{J}^{\circ}(\mu)\right) & =\mathbb{R}, \quad \mu>1, \quad \sigma_{\text {a.c. }}\left(\mathbf{J}^{\circ}(1)\right)=[0, \infty) ; \\
\mathfrak{m}_{\text {a.c. }}\left(\lambda ; \mathbf{J}^{\circ}(\mu)\right) & =1 \quad \text { for a.e. } \lambda \in \sigma\left(\mathbf{J}^{\circ}(\mu)\right) .
\end{aligned}
$$

The proof, given in [11, Appendix 1], is based on the theory of "subordinate solutions" of second-order differential equations, as presented by Gilbert and Pearson in [3], or, more precisely, on the version of that theory for Jacobi matrices, developed in [8].

The following theorem is one of the main results of the present paper. The statements of Theorems 3.1-3.3 about the absolutely continuous spectrum of $\mathbf{A}_{\alpha}$ are direct consequences of this theorem.

Theorem 3.6. Let $\alpha>0$, and let $\mu=\mu(\alpha)=d(o)(\alpha \sqrt{2})^{-1}$. Then the absolutely continuous spectrum of the operator $\mathbf{A}_{\alpha}$ and its multiplicity function are given by the formulas

$$
\begin{aligned}
\sigma_{\text {a.c. }}\left(\mathbf{A}_{\alpha}\right) & =\sigma_{\text {a.c. }}\left(\mathbf{A}_{0}\right) \cup \sigma_{\text {a.c. }}\left(\mathbf{J}^{\circ}(\mu)\right) \\
\mathfrak{m}_{\text {a.c. }}\left(\lambda ; \mathbf{A}_{\alpha}\right) & =\mathfrak{m}_{\text {a.c. }}\left(\lambda ; \mathbf{A}_{0}\right)+\mathfrak{m}_{\text {a.c. }}\left(\lambda ; \mathbf{J}^{\circ}(\mu)\right) .
\end{aligned}
$$

The proof of Theorem 3.6 is the most difficult technical part of the paper. The proof is given in $\S \S 6-8$. Statements 2) and 3) of Theorem 3.1 are proved in $\S 10$. Statement 2) of Theorem 3.2 is proved in Subsection 9.2. Statement 3) of Theorem 3.2 and statement 2 ) of Theorem 3.3 are proved in $\S 11$. We do not present the proof of Proposition 3.4, but give some references. The positive definiteness of the operator $\mathbf{A}_{\alpha}$ for $\Gamma=\Gamma_{d}$ and $\alpha \sqrt{2}<d$ was proved in [14]. In [11] it was shown that the point spectrum is empty in the case where $\alpha \sqrt{2} \geq d$ and $d=2$; for $d \neq 2$ the proof is much the same.

\section{§4. Auxiliary material}

Here we collect the technical facts to be used in what follows.

1. On the Green function of the Laplacian on a graph. A function $g(\zeta ; x, o)$ on $\Gamma \times \Gamma$, depending on the complex variable $\zeta \in \mathbb{C} \backslash(-\infty, 0]$, will play a very important role in what follows. As usual, the point $o$ is fixed. It is convenient to assume that $o \in \mathcal{V}$, but we do not exclude the case where $d(o)=2$. The function $g(\zeta ; ., o)$ is defined as the $H^{1}(\Gamma)$-solution of the problem

$$
\begin{aligned}
& -g^{\prime \prime}(x)+\zeta g(x)=0, \quad x \notin \mathcal{V} ; \\
& {\left[g^{\prime}\right](x)=0, \quad x \in \mathcal{V}, \quad x \neq o ; \quad g(o)=1 .}
\end{aligned}
$$

Such a solution exists and is unique because $-\zeta \notin \sigma(-\Delta)$. Clearly, up to normalization, $g(\zeta ; x, o)$ is the Green function for the equation $-\Delta u+\zeta u=f$ on $\Gamma$. The normalization coefficient is

$$
r(\zeta ; o):=-\left.\left[g_{x}^{\prime}(\zeta ; ., o)\right]\right|_{x=o} .
$$

Thus, in particular,

$$
-\Delta u+\zeta u=f \Longrightarrow u(o)=r(\zeta ; o)^{-1} \int_{\Gamma} g(\zeta ; x, o) f(x) d x .
$$


The following identity is fulfilled for any $u \in H^{1}(\Gamma)$ :

$$
\int_{\Gamma}\left(u_{x}^{\prime}(x) g_{x}^{\prime}(\zeta ; x, o)+\zeta u(x) g(\zeta ; x, o)\right) d x=r(\zeta ; o) u(o) .
$$

Assume that $\zeta=k^{2}$ is a real positive number. We put $u(x)=g\left(k^{2} ; x, o\right)$ in (4.3). Then

$$
\int_{\Gamma}\left(g_{x}^{\prime 2}\left(k^{2} ; x, o\right)+k^{2} g^{2}\left(k^{2} ; x, o\right)\right) d x=r\left(k^{2}, o\right) .
$$

If $\Gamma=\Gamma_{d}$ and $o$ is the only vertex of the graph $\Gamma_{d}$, then the function $g(\zeta ; x, o)$ can be computed easily. Namely,

$$
g(\zeta ; x, o)=e^{-\sqrt{\zeta} \rho(x, o)}, \quad \Gamma=\Gamma_{d},
$$

where the branch of the square root for which $\operatorname{Re} \sqrt{\zeta}>0$ is chosen. From (4.5) we obtain

$$
\begin{aligned}
r(\zeta ; o) & =d \sqrt{\zeta}, \quad \Gamma=\Gamma_{d} ; \\
2 k\|g(\zeta ; ., o)\|_{L^{2}(\Gamma)}^{2} & =d, \quad k=\operatorname{Re} \sqrt{\zeta}, \quad \Gamma=\Gamma_{d} .
\end{aligned}
$$

We shall show that, up to an exponentially small correction, identity (4.6) (with $d(o)$ in place of $d$ ) remains valid in the general case, i.e., for an arbitrary graph $\Gamma \in \mathfrak{G}$ and any point $o \in \Gamma$. Here we assume that $\zeta$ tends to infinity in a neighborhood of the positive half-axis. Under the same conditions, identity (4.7) should be replaced by a uniform two-sided estimate.

First, consider the case where $\zeta \in \mathbb{R}_{+}$.

Lemma 4.1. Let $\Gamma \in \mathfrak{G}$, and let $o \in \Gamma$ be an arbitrary point. If $\zeta=k^{2}>0$, then

$$
r\left(k^{2} ; o\right)=k d(o)\left(1+O\left(e^{-2 k \varepsilon(\Gamma, o)}\right)\right),
$$

and there exist positive numbers $M_{1}, M_{2}$ such that

$$
M_{1} d(o) \leq k\left\|g\left(k^{2} ; ., o\right)\right\|_{L^{2}(\Gamma)}^{2} \leq M_{2} d(o) .
$$

Both estimates are uniform in $k$ for $k \geq k_{0}>0$.

The next result shows that for nonreal $\zeta$, inequalities (4.9) stay the same. The estimate of $r(\zeta ; o)-d(o) \sqrt{\zeta}$ becomes slightly weaker, but still suffices for our purposes.

Lemma 4.2. Let $\Gamma \in \mathfrak{G}$, and let $o \in \Gamma$. Assume that $|\operatorname{Im} \zeta| \leq c$, where $c>0$ is arbitrary. Then

$$
r(\zeta ; o)=\sqrt{\zeta} d(o)+O\left(k^{-1} e^{-k \varepsilon(\Gamma, o)}\right), \quad k=\operatorname{Re} \sqrt{\zeta} \rightarrow+\infty,
$$

and there exist positive numbers $M_{1}, M_{2}$ depending on $c$ such that

$$
M_{1} d(o) \leq k\|g(\zeta ; ., o)\|_{L^{2}(\Gamma)}^{2} \leq M_{2} d(o) .
$$

Both estimates are uniform in $k=\operatorname{Re} \sqrt{\zeta}$ for $k \geq 1$.

The proof of these lemmas is not difficult but cumbersome. We postpone it till $\S 13$.

2. An auxiliary recurrence system. The matrix $\mathbf{J}(\Lambda ; \mu)$. In the sequel, we shall often come across the equation

$$
\mathbf{A}_{\alpha} W-\Lambda W=0,
$$

where $\Lambda$ is the spectral parameter, $\Lambda \notin[1 / 2, \infty)$. If an element $W \sim\left\{w_{n}\right\}$ satisfies (4.12), then, by (2.5),

$$
-w_{n}^{\prime \prime}(x)+(n+1 / 2-\Lambda) w_{n}(x)=0, \quad x \notin \mathcal{V}, \quad n \in \mathbb{N}_{0},
$$


and conditions (2.6) are fulfilled. Since $n+1 / 2-\Lambda \notin(-\infty, 0]$ for any $n$, the function $w_{n}(x)$ is proportional to $g(n+1 / 2-\Lambda ; x, o)$. Put

$$
\eta_{n}(\Lambda ; x)=(n+1 / 2)^{1 / 4} g(n+1 / 2-\Lambda ; x, o) .
$$

The functions (4.14) are more convenient to deal with than $g(n+1 / 2-\Lambda ; x, o)$ because (4.11) implies

$$
M_{1} d(o) \leq\left\|\eta_{n}(\Lambda ; .)\right\|_{L^{2}(\Gamma)}^{2} \leq M_{2} d(o), \quad n \in \mathbb{N}_{0}, \quad M_{1}>0 .
$$

This estimate is uniform in $\Lambda$ on any compact set in $\mathbb{C} \backslash[1 / 2, \infty)$. We denote

$$
r_{n}(\Lambda)=r(n+1 / 2-\Lambda ; o) .
$$

The quantities $r_{n}(\Lambda)$ are well defined for any $n \in \mathbb{N}_{0}$.

Every function $w_{n}$ can be written as

$$
w_{n}(x)=C_{n} \eta_{n}(\Lambda ; x) \text {. }
$$

To define the coefficients $C_{n}$, we use conditions (2.7). After some elementary transformations, we get the recurrence system of linear equations

$$
q_{n+1} C_{n+1}+2 \mu \frac{r_{n}(\Lambda)}{d(o)} \sqrt{n+1 / 2} C_{n}+q_{n} C_{n-1}=0, \quad \mu=d(o)(\alpha \sqrt{2})^{-1},
$$

where the coefficients $q_{n}$ are as in (3.4). The solution of (4.18) is unique if we fix $C_{0}$. By (4.15), we have

$$
W \sim\left\{C_{n} \eta_{n}(\Lambda, .)\right\} \in \mathfrak{H} \Longleftrightarrow\left\{C_{n}\right\} \in \ell^{2} .
$$

System (4.18) is generated by the Jacobi matrix

$$
\mathbf{J}(\Lambda ; \mu)=\mathbf{J}\left(\left\{2 \mu y_{n}(\Lambda)\right\},\left\{q_{n}\right\}\right)
$$

where

$$
y_{n}(\Lambda)=\frac{r_{n}(\Lambda)}{d(o)} \sqrt{n+1 / 2} .
$$

Along with the matrix $\mathbf{J}^{\circ}(\mu)$ defined in (3.3), the matrix $\mathbf{J}(\Lambda ; \mu)$ plays a crucial role in the investigation of the spectrum of the operator $\mathbf{A}_{\alpha}$. In particular, this matrix is involved in the important formula (6.4) for the difference of the resolvents of $\mathbf{A}_{\alpha}$ and $\mathbf{A}_{0}$.

3. The Birkhoff-Adams theorem. To investigate system (4.18), we use the BirkhoffAdams theorem. This classical result concerns recurrence systems of the form

$$
C(n+1)+p_{1}(n) C(n)+p_{2}(n) C(n-1)=0
$$

and describes the asymptotic behavior of two linearly independent solutions of the system. It is assumed that the functions $p_{1}(n)$ and $p_{2}(n)$ possess asymptotic expansions

$$
p_{1}(n) \sim \sum_{j=0}^{\infty} a_{j} n^{-j}, \quad p_{2}(n) \sim \sum_{j=0}^{\infty} b_{j} n^{-j}, \quad b_{0} \neq 0 .
$$

The following statement is borrowed from Theorem 8.16 in [5]. We cite only its part that is used in the present paper. Also, we correct an obvious misprint in [5, formula (8.6.7)].

Proposition 4.3 (Birkhoff-Adams theorem). Let $\lambda_{ \pm}$be the zeros of the quadratic equation $\lambda^{2}+a_{0} \lambda+b_{0}=0$.

a) If $\lambda_{+} \neq \lambda_{-}$, then system (4.22) has two linearly independent solutions $\left\{C^{ \pm}(n)\right\}$ with the asymptotic behavior

$$
C^{ \pm}(n) \sim \lambda_{ \pm}^{n} n^{d_{ \pm}}, \quad d_{ \pm}=\frac{a_{1} \lambda_{ \pm}+b_{1}}{a_{0} \lambda_{ \pm}+2 b_{0}}
$$


b) If $\lambda_{+}=\lambda_{-}=\lambda$, but $2 b_{1} \neq a_{0} a_{1}$, then system (4.22) has two linearly independent solutions $\left\{C^{ \pm}(n)\right\}$ with the asymptotic behavior

$$
C^{ \pm}(n) \sim \lambda^{n} e^{ \pm \delta \sqrt{n}} n^{\varkappa}, \quad \delta=2 \sqrt{\frac{a_{0} a_{1}-2 b_{1}}{2 b_{0}}}, \quad \varkappa=\frac{1}{4}+\frac{b_{1}}{2 b_{0}} .
$$

We also need an identity satisfied by any solution of a recurrence system of the form

$$
Q_{n+1} C_{n+1}+P_{n} C_{n}+Q_{n} C_{n-1}=0, \quad n \in \mathbb{N}_{0},
$$

where all $Q_{n}$ are real and $Q_{0}=0$. This identity looks like this:

$$
\sum_{n=0}^{N}\left|C_{n}\right|^{2} \operatorname{Im} P_{n}=-Q_{N+1} \operatorname{Im}\left(C_{N+1} \overline{C_{N}}\right), \quad N \in \mathbb{N} .
$$

The proof is straightforward, and we omit it.

We return to the system (4.18). Rewriting it in the form (4.22) and using the asymptotic formula (4.10) for $r(\zeta ; o)$, we see that for $\Lambda \notin[1 / 2, \infty)$ the coefficients of the new system admit asymptotic expansions in powers of $n^{-1}$. We only need their first two terms:

$$
p_{1}(n) \sim 2 \mu\left(1-\frac{1+\Lambda}{2 n}\right), \quad p_{2}(n) \sim 1-\frac{1}{n} .
$$

Lemma 4.4. For any $\Lambda \notin[1 / 2, \infty)$, system (4.18) has two linearly independent solutions with the following asymptotic behavior:

a) if $\mu \neq 1$, then

$$
C_{n}^{ \pm} \sim\left(\lambda_{+}\right)^{ \pm n} n^{d_{ \pm}}, \quad d_{ \pm}=-\frac{1}{2} \mp \frac{\Lambda \mu}{2 \sqrt{\mu^{2}-1}}, \quad \mu \neq 1 ;
$$

b) if $\mu=1$ and $\Lambda \neq 0$, then

$$
C_{n}^{ \pm} \sim(-1)^{n} e^{ \pm 2 \sqrt{-\Lambda n}} n^{-1 / 4}
$$

Proof. The equation for $\lambda$ takes the form $\lambda^{2}+2 \mu \lambda+1=0$. Its zeros $\lambda_{ \pm}=-\mu \pm \sqrt{\mu^{2}-1}$ do not depend on $\Lambda$. Obviously, $\lambda_{-}=\lambda_{+}^{-1}$. Now, both statements of the lemma follow from Proposition 4.3.

4. Concluding remarks to $\S 4$. The technical results of the present section will be used later on to prove that the operator $\mathbf{A}_{\alpha}$ is selfadjoint as well as to analyze the absolutely continuous and the point spectrum of the operator. The problem is reduced to an investigation of the homogeneous equation (4.12) for some $\Lambda$ or of the corresponding heterogeneous equation. Every time we use Lemma 4.4, i.e., in essence, the BirkhoffAdams theorem. It is important that the result only depends on the asymptotic behavior of the sequence (4.16). In its turn, this asymptotic behavior is completely determined by the structure of the set $\mathcal{S}(o)$ and does not depend on other characteristics of the graph (a sort of a "local principle"). This explains the character of the main results of the paper: the value of the parameter $\alpha$ where the phase transition happens depends on $d(o)$ only. Again, only this parameter is involved in the formulation of Theorem 3.6 and of statement 3) in Theorem 3.1.

\section{$\S 5$. Proof of Theorem 2.1}

Having Lemma 4.4 at hand, it is not difficult to prove Theorem 2.1.

Along with the set $\mathcal{D}_{\alpha}$ described in the theorem, consider its subset $\mathcal{D}_{\alpha}^{0}$ generated by the elements $U \sim\left\{u_{n}\right\}$ with finitely many nonzero functions $u_{n}$. The set $\mathcal{D}_{\alpha}^{0}$ is dense in $\mathfrak{H}$, and the operator $\mathbf{A}_{\alpha}^{0}:=\mathcal{A} \backslash \mathcal{D}_{\alpha}^{0}$ is symmetric. It is easy to show that

$$
\mathbf{A}_{\alpha}=\left(\mathbf{A}_{\alpha}^{0}\right)^{*} .
$$


For the case where $\Gamma$ is a star graph and $o$ is the central vertex, the proof of (5.1) was given in [14. Later, the proof was repeated in [11 (for $\Gamma=\mathbb{R})$. For the general graphs the proof is much the same and we omit it.

As soon as (5.1) is established, it remains to verify that the deficiency indices of the operator $\mathbf{A}_{\alpha}^{0}$ are equal to zero. The coefficients in the differential expression (2.1) and in conditions (2.2) are real. Therefore, it suffices to show that the equation

$$
\mathbf{A}_{\alpha} W=i W
$$

has only the trivial solution in $\mathfrak{H}$. Equation (5.2) coincides with equation (4.12) for $\Lambda=i$ and reduces to system (4.18). Thus, we can apply Lemma 4.4. First, let $\alpha \sqrt{2}>d(o)$. Then $\mu<1$ and the absolute value of the zeros $\lambda_{ \pm}$is equal to 1 . In accordance with (4.25), we have

$$
\left|C_{n}^{ \pm}\right| \sim n^{d_{ \pm}} .
$$

Recalling (4.19), we see that only the sequence $C_{n}^{+}$can produce $W \in \mathfrak{H}$. Suppose this is the case. Observe that (4.18) is a system of the form (4.23) with $Q_{n}=q_{n}$ and $P_{n}=2 \mu y_{n}(\Lambda)$. Identity (4.24) gives

$$
\sum_{n=0}^{N}\left|C_{n}^{+}\right|^{2} \operatorname{Im} P_{n}=-\alpha \sqrt{N+1} \operatorname{Im}\left(C_{N+1}^{+} \overline{C_{N}^{+}}\right) .
$$

By (5.3), the right-hand side tends to zero as $N \rightarrow \infty$. Since $\operatorname{Im} P_{n}>0$ for all $n$, it follows that $C_{n}^{+}=0$ for all $n$. Thus, equation (5.2) has only the trivial solution in $\mathfrak{H}$. So, for $\alpha \sqrt{2}>d(o)$ the operator $\mathbf{A}_{\alpha}$ is selfadjoint.

For $\alpha \sqrt{2}<d(o)$, i.e., for $\mu>1$, the argument is similar. In this case one of the basis solutions $\left\{C_{n}^{ \pm}\right\}$of (4.18) grows exponentially, and the other decays exponentially. Only the latter can produce the solution $W \in \mathfrak{H}$ of equation (5.2). As in the case of $\mu<1$, we use identity (4.24) to prove that such a solution is equal to zero.

If $\alpha \sqrt{2}=d(o)$, i.e., $\mu=1$, then $\lambda_{+}=\lambda_{-}=-1$. In accordance with (4.26), the system has two solutions with the asymptotic behavior $C_{n}^{ \pm} \sim e^{ \pm(1-i) \sqrt{2 n}}$. Only $\left\{C_{n}^{-}\right\}$ can produce the solution $W \in \mathfrak{H}$. Applying identity (4.24) once again, we see that this solution is equal to zero.

We have proved that the operator $\mathbf{A}_{\alpha}$ is selfadjoint for all $\alpha>0$. For $\alpha=0$, selfadjointness is clear from the construction.

\section{§6. Absolutely continuous spectrum: Preparatory results}

1. A formula for the difference of resolvents. Technically, the study of the absolutely continuous spectrum is the most difficult part of the paper. We start with deducing a convenient representation (see (6.4) below) for the difference of resolvents

$$
\mathbf{Q}_{\alpha}(\Lambda):=\left(\mathbf{A}_{\alpha}-\Lambda\right)^{-1}-\left(\mathbf{A}_{0}-\Lambda\right)^{-1}, \quad \Lambda \notin \mathbb{R} .
$$

This representation involves the Jacobi matrix $\mathbf{J}(\Lambda ; \mu)$ (see (4.20)) and the diagonal operator

$$
\mathbf{Y}(\Lambda)=\operatorname{diag}\left\{y_{n}(\Lambda)\right\}
$$

(see (4.21)). The operator $\mathbf{Y}(\Lambda)$ is unbounded in $\ell^{2}$ and is closed on its natural domain. It is clear that for $\Lambda \notin[1 / 2, \infty)$ the operator $\mathbf{Y}(\Lambda)^{-1}$ is bounded and the operator-valued function $\mathbf{Y}(\Lambda)^{-1}$ is analytic.

The representation (6.4) also involves the operator

$$
\mathbf{T}(\Lambda): \ell^{2}\left(\mathbb{N}_{0}\right) \rightarrow \mathfrak{H}, \quad \mathbf{T}(\Lambda)\left\{C_{n}\right\}=\left\{C_{n} \eta_{n}(\Lambda ; x)\right\} .
$$


By (4.15), the operators $\mathbf{T}(\Lambda)$ and $\mathbf{T}(\Lambda)^{-1}$ are bounded for $\Lambda \notin[1 / 2, \infty)$. The action of the adjoint operator $\mathbf{T}(\Lambda)^{*}$ on the element $F \sim\left\{f_{n}\right\} \in \mathfrak{H}$ is given by

$$
\mathbf{T}(\Lambda)^{*} F \sim\left\{I_{n}\left(\bar{\Lambda} ; f_{n}\right)\right\},
$$

where for any $\Lambda \notin[1 / 2, \infty)$ and $f \in L^{2}(\Gamma)$ we use the notation

$$
I_{n}(\Lambda ; f)=\int_{\Gamma} \eta_{n}(\Lambda ; x) f(x) d x
$$

The operator-valued functions $\mathbf{T}(\Lambda)$ and $\mathbf{T}(\bar{\Lambda})^{*}$ are analytic in $\mathbb{C} \backslash[1 / 2, \infty)$.

Theorem 6.1. For any $\Lambda \notin \mathbb{R}$ we have

$$
\left(\mathbf{A}_{\alpha}-\Lambda\right)^{-1}-\left(\mathbf{A}_{0}-\Lambda\right)^{-1}=\mathbf{T}(\Lambda)\left(\mu \mathbf{J}(\Lambda ; \mu)^{-1}-(d(o) \mathbf{Y}(\Lambda))^{-1}\right) \mathbf{T}(\bar{\Lambda})^{*}
$$

where $\mu=\mu(\alpha)=d(o)(\alpha \sqrt{2})^{-1}$.

Proof. Given an element $F \sim\left\{f_{n}\right\} \in \mathfrak{H}$, for any $\alpha \geq 0$ we define

$$
U_{\alpha} \sim\left\{u_{\alpha, n}\right\}=\left(\mathbf{A}_{\alpha}-\Lambda\right)^{-1} F ; \quad W:=U_{\alpha}-U_{0} \sim\left\{w_{n}\right\} .
$$

Every function $u_{0, n}$ solves the problem $-\Delta u+(n+1 / 2-\Lambda) u=f_{n}$. Therefore, by (4.2),

$$
r_{n}(\Lambda) u_{0, n}(o)=\int_{\Gamma} g(n+1 / 2-\Lambda ; x, o) f_{n}(x) d x=(n+1 / 2)^{-1 / 4} I_{n}\left(\Lambda ; f_{n}\right) .
$$

The functions $w_{n}$ are continuous on $\Gamma$. They satisfy the homogeneous equation (4.13) and conditions (2.6). Therefore, $w_{n}=C_{n} \eta_{n}(\Lambda,$.$) (see (4.17)). This means that$

$$
\mathbf{Q}_{\alpha}(\Lambda)=\mathbf{T}(\Lambda)\left\{C_{n}\right\}
$$

Thus, we only need to find the coefficients $\left\{C_{n}\right\}$. To this end, we use conditions (2.7) for the functions $u_{\alpha, n}$.

Observe that $\left[u_{0, n}^{\prime}\right](o)=0$, whence $\left[w_{n}^{\prime}\right](o)=\left[u_{\alpha, n}^{\prime}\right](o)$. Using (2.7) and (4.1), we obtain

$$
\begin{aligned}
-C_{n}(n & +1 / 2)^{1 / 4} r_{n}(\Lambda)=\left[w_{n}^{\prime}\right](o)=\left[u_{\alpha, n}^{\prime}\right](o) \\
= & \frac{\alpha}{\sqrt{2}}\left(\sqrt{n+1} u_{\alpha, n+1}(o)+\sqrt{n} u_{\alpha, n-1}(o)\right) .
\end{aligned}
$$

Using the definition of $W$ and equation (4.14), we get

$$
u_{\alpha, n}(o)=u_{0, n}(o)+w_{n}(o)=u_{0, n}(o)+C_{n}(n+1 / 2)^{1 / 4} .
$$

Put $X_{n}=(n+1 / 2)^{-1 / 4} u_{\alpha, n}(o)$. Then $X_{n}=C_{n}+(n+1 / 2)^{-1 / 4} u_{0, n}(o)$. Taking (6.5) into account, we reduce (6.7) to a nonhomogeneous system with the matrix $\mathbf{J}(\Lambda ; \mu)$ :

$$
q_{n+1} X_{n+1}+2 \mu y_{n}(\Lambda) X_{n}+q_{n} X_{n-1}=\sqrt{2} \alpha^{-1} I_{n}\left(\bar{\Lambda}, f_{n}\right) .
$$

Combined with (6.3), this shows that $\left\{X_{n}\right\}=\sqrt{2} \alpha^{-1} \mathbf{J}(\Lambda ; \mu)^{-1} \mathbf{T}(\bar{\Lambda})^{*} F$.

Identity (6.5) can be rewritten in the form

$$
\left\{(n+1 / 2)^{-1 / 4} u_{0, n}(o)\right\}=(d(o) \mathbf{Y}(\Lambda))^{-1} \mathbf{T}(\bar{\Lambda})^{*} F .
$$

Now, the claim follows from the last two identities and (6.6).

At this point, we discuss relation (6.4) from the viewpoint of the theory of extensions. For any $\alpha \geq 0$, the operator $\mathbf{A}_{\alpha}$ is a selfadjoint extension of the symmetric operator $\widehat{\mathbf{A}}:=\mathcal{A} \uparrow \widehat{\mathcal{D}}$, where the set $\widehat{\mathcal{D}}$ is formed by the elements $U \sim\left\{u_{n}\right\} \in \mathcal{D}_{\alpha}$ whose support misses the point $x=o$. Clearly, this definition does not depend on $\alpha$.

The operator on the right in (6.4) acts nontrivially only between $\operatorname{Ran} T(\bar{\Lambda})$ and $\operatorname{Ran} T(\Lambda)$. Moreover, it is easy to verify that

$$
\operatorname{Ran} T(\bar{\Lambda}) \subset \operatorname{Ker}\left(\widehat{\mathbf{A}}^{*}-\bar{\Lambda}\right), \quad \operatorname{Ran} T(\Lambda) \subset \operatorname{Ker}\left(\widehat{\mathbf{A}}^{*}-\Lambda\right) .
$$


Therefore, formula (6.4) agrees with the general theory, which states that the difference of the resolvents of any two selfadjoint extensions of the operator $\widehat{\mathbf{A}}$ can act nontrivially only between the subspaces occurring on the right-hand sides in (6.8).

2. Outline of the proof of Theorem 3.6. We shall rely upon two well-known facts concerning general selfadjoint operators $\mathbf{A}$ in the Hilbert space $\mathcal{H}$. The first fact is elementary. Let $\mathbf{A}=\mathbf{A}^{*}$, and let $\mathfrak{m}_{\text {a.c. }}(\lambda ; \mathbf{A})>0$ for a.e. $\lambda \in I$, where $I \subset \mathbb{R}$ is an interval. Then $I \subset \sigma_{\text {a.c. }}(\mathbf{A})$. If $\mathfrak{m}_{\text {a.c. }}(\lambda ; \mathbf{A})=0$ for a.e. $\lambda \in I$, then $I \cap \sigma_{\text {a.c. }}(\mathbf{A})=\varnothing$. Thus, it suffices to prove identity (3.6) in Theorem 3.6. Identity (3.5) will follow automatically.

The second fact is not so obvious. We use a relationship between the absolutely continuous spectrum of the operator $\mathbf{A}$ and the jump of the bordered resolvent under crossing the real axis.

Let $\Omega \subset \mathbb{C}$ be a domain symmetric with respect to the real axis and containing an interval $I \subset \mathbb{R}$. Let $\mathbf{Z}(\Lambda)$ be an operator-valued function analytic in $\Omega \cap \mathbb{C}_{+}$and $\Omega \cap \mathbb{C}_{-}$. The jump of $\mathbf{Z}(\Lambda)$ at a point $\lambda \in I$ is defined as the strong nontangential limit

$$
[\mathbf{Z}](\lambda):=\underset{\Lambda \rightarrow \lambda+i 0}{(\mathrm{~s})-\lim }(\mathbf{Z}(\Lambda)-\mathbf{Z}(\bar{\Lambda}))
$$

(if this limit exists). Earlier we have used the notation [.] for the expression occurring in Kirchhoff's condition (see (1.1) and (1.2)). This will not cause confusion, because the meaning of the notation will always be clear from the context.

Now, let $\mathbf{A}$ be a selfadjoint operator in $\mathcal{H}$ and let $\mathbf{Z}(\Lambda)=\mathbf{Z}_{\mathbf{G}}(\Lambda)$ be its bordered resolvent:

$$
\mathbf{Z}_{\mathbf{G}}(\Lambda)=G(\mathbf{A}-\Lambda)^{-1} G^{*}
$$

Here $\mathbf{G}$ is a bounded operator in $\mathcal{H}$. It was shown in [2] (see also [16, Theorem 6.1.9]) that for any $\mathbf{A}=\mathbf{A}^{*}$ and any $\mathbf{G} \in \mathfrak{S}_{2}$ the jump of the function $\mathbf{Z}_{\mathbf{G}}(\Lambda)$ exists for a.e. $\lambda \in \mathbb{R}$ and is a trace class operator $\left(\left[\mathbf{Z}_{\mathbf{G}}\right](\lambda) \in \mathfrak{S}_{1}\right)$. Certainly, for a fixed operator $\mathbf{A}$ the jump can exist for a wider class of borderings.

The following statement is true.

Theorem 6.2. Let $\mathbf{A}$ be a selfadjoint operator in the Hilbert space $\mathcal{H}$, and let $\Delta \subset \mathbb{R}$ be a Borel set. If $\mathbf{G}$ is a bounded operator in $\mathcal{H}$ such that its kernel is trivial and the jump

$$
\mathbf{V}_{\mathbf{A}, \mathbf{G}}(\lambda)=\underset{\Lambda \rightarrow \lambda+i 0}{(\mathrm{~s})-\lim _{0}} \mathbf{G}\left((\mathbf{A}-\Lambda)^{-1}-(\mathbf{A}-\bar{\Lambda})^{-1}\right) \mathbf{G}^{*}
$$

is well defined for a.e. $\lambda \in \Delta$, then

$$
\mathfrak{m}_{\text {a.c. }}(\lambda ; \mathbf{A})=\operatorname{rank} \mathbf{V}_{\mathbf{A}, \mathbf{G}}(\lambda) \quad \text { for a.e. } \lambda \in \Delta .
$$

The proof can be found in [11, Appendix 2]. This theorem implies that if $\operatorname{rank} \mathbf{V}_{\mathbf{A}, \mathbf{G}}(\lambda)$ is positive a.e. on an interval $I \subset \mathbb{R}$, then $I \subset \sigma_{\text {a.c. }}(\mathbf{A})$.

The next step of the plan consists in finding an appropriate bordering $\mathbf{G}$ and using formula (6.4) to express the jump $\mathbf{V}_{\mathbf{A}_{\alpha}, \mathbf{G}}(\lambda)$ in terms of $\mathbf{V}_{\mathbf{A}_{0}, \mathbf{G}}(\lambda)$ and the jump of the operator-valued function on the right-hand side of identity (6.4), with the same bordering.

$$
\S 7 \text {. Operators } \mathbf{J}^{\circ}(\mu) \text { And } \mathbf{J}(\Lambda ; \mu)
$$

1. The operator-valued function $\mathbf{J}(\Lambda ; \mu)$. The matrix $\mathbf{J}(\Lambda ; \mu)$ is involved in identity (6.4), while the matrix $\mathbf{J}^{\circ}(\mu)$ occurs in the formulation of Theorem 3.6 (see (3.3)). The matrix

$$
\mathbf{J}(\Lambda ; \mu)-\left(\mathbf{J}^{\circ}(\mu)-\mu \Lambda\right)=\mu \operatorname{diag}\left\{2 y_{n}(\Lambda)-(2 n+1-\Lambda)\right\}
$$

is diagonal. By (4.21) and (4.10), we have

$$
\psi_{n}(\Lambda):=2 y_{n}(\Lambda)-(2 n+1-\Lambda)=O\left(n^{-1}\right) .
$$


Therefore, the operator (7.1) is compact in $\ell^{2}$. This allows us to investigate the properties of the operator $\mathbf{J}(\Lambda ; \mu)$ for a fixed $\Lambda$, as well as the properties of the operator-valued function $\mathbf{J}(\Lambda ; \mu)$ for a fixed value of $\mu$.

First, the compactness of the operator (7.1) implies that the operator $\mathbf{J}(\Lambda ; \mu)$ is closed on the domain of the selfadjoint operator $\mathbf{J}^{\circ}(\mu)$. Next, $\operatorname{Im} \mathbf{J}(\Lambda ; \mu)=2 \mu \operatorname{Im} \mathbf{Y}(\Lambda)$. It follows that

$$
\operatorname{sgn}(\operatorname{Im} \mathbf{J}(\Lambda ; \mu))=-\operatorname{sgn} \Lambda .
$$

Therefore, for $\Lambda \notin \mathbb{R}$ the matrix $\mathbf{J}(\Lambda ; \mu)$ is boundedly invertible in $\ell^{2}$. Moreover, the operator-valued function $\mathbf{J}(\Lambda ; \mu)^{-1}$ is analytic with respect to $\Lambda$ in each of the half-planes $\mathbb{C}_{ \pm}=\{\Lambda \in \mathbb{C}: \pm \operatorname{Im} \Lambda>0\}$.

The definition of the sequence $\left\{y_{n}(\Lambda)\right\}$ (see (4.21)) and the asymptotics (4.10) show that there exists a constant $c>0$ such that

$$
\left|\operatorname{Im} y_{n}( \pm i \tau)\right| \geq c \tau^{1 / 2}, \quad \tau \geq 1
$$

Combined with a well-known estimate for dissipative operators (see [4, Theorem IV.2.1]), this implies the following useful inequality:

$$
\left\|\mathbf{J}( \pm i \tau ; \mu)^{-1}\right\| \leq\left(2 \mu c \tau^{1 / 2}\right)^{-1}, \quad \tau \geq 1 .
$$

2. Behavior of $\mathbf{J}(\Lambda ; \mu)^{-1}$ and $\left(\mathbf{J}^{\circ}(\mu)-\mu \Lambda\right)^{-1}$ as $\operatorname{Im} \Lambda \rightarrow 0$. In general, the limits of these functions as $\Lambda$ approaches the real axis do not exist. The situation changes if we consider appropriate borderings.

We define a selfadjoint operator in $\ell^{2}$ by

$$
\widetilde{\mathbf{G}}_{\gamma}=\operatorname{diag}\left\{(n+1 / 2)^{-\gamma}\right\}, \quad \gamma>0,
$$

and consider the operator-valued functions

$$
\widetilde{\mathbf{Z}_{\gamma}}(\Lambda ; \mu)=\widetilde{\mathbf{G}}_{\gamma} \mathbf{J}(\Lambda ; \mu)^{-1} \widetilde{\mathbf{G}}_{\gamma}, \quad \widetilde{\mathbf{Z}_{\gamma}^{\circ}}(\Lambda ; \mu)=\widetilde{\mathbf{G}}_{\gamma}\left(\mathbf{J}^{\circ}(\mu)-\mu \Lambda\right)^{-1} \widetilde{\mathbf{G}}_{\gamma} .
$$

Lemma 7.1. Let $\gamma>1 / 4$. Then for any $\Lambda \notin \mathbb{R}$ and $\mu>0$ the operators $\widetilde{\mathbf{Z}_{\gamma}}(\Lambda ; \mu)$ and $\widetilde{\mathbf{Z}_{\gamma}^{\circ}}(\Lambda ; \mu)$ are of trace class, and the strong nontangential limits $\widetilde{\mathbf{Z}_{\gamma}}(\lambda \pm i 0 ; \mu)$ and $\widetilde{\mathbf{Z}_{\gamma}^{\circ}}(\lambda \pm i 0 ; \mu)$ exist for a.e. $\lambda \in \mathbb{R}$.

Note that $\mathbf{G}_{\gamma} \in \mathfrak{S}_{2}$ only for $\gamma>1 / 2$. Therefore, the above result does not follow from the general theory even for the operator $\widetilde{\mathbf{Z}_{\gamma}^{\circ}}(\Lambda ; \mu)$ when we deal with the bordered resolvent of the operator $\mathbf{J}^{\circ}(\mu)$.

Proof of the lemma. We discuss in detail the harder case of the function $\widetilde{\mathbf{Z}_{\gamma}}(\Lambda ; \mu)$. For definiteness, let $\operatorname{Im} \Lambda>0$. The operator-valued function under consideration is analytic with respect to $\Lambda$ and its values are dissipative operators. The results of [11] show that this function admits the representation

$$
\widetilde{\mathbf{Z}_{\gamma}}(\Lambda ; \mu)=A+B \Lambda+R^{*}(I+\Lambda \mathcal{L})(\mathcal{L}-\Lambda)^{-1} R, \quad \operatorname{Im} \Lambda>0,
$$

where $\mathcal{L}$ is a selfadjoint operator in some auxiliary Hilbert space $\mathcal{H}$, the operators $A, B$ are selfadjoint in $\ell^{2}$, and $R$ is a bounded operator from $\ell^{2}$ into $\mathcal{H}$. Our notation for these operators does not reflect their dependence on $\mu$, because the value of $\mu$ is fixed. Estimate (7.3) implies that $B=0$. Choosing $\Lambda=i$ in (7.5), we see that

$$
\widetilde{\mathbf{Z}_{\gamma}}(i ; \mu)=A+i R^{*} R \text {. }
$$

Next, consider the matrix $\left\{g_{n, k}\right\}:=\mathbf{J}(i ; \mu)^{-1}$. This matrix is symmetric (i.e., $g_{n, k}=$ $\left.g_{k, n}\right)$. For $k \geq n$ its elements can be expressed via solutions of the homogeneous system with the matrix $\mathbf{J}(i ; \mu)$; this is system (4.18) with $\Lambda=i$. By (4.25) and (4.26), for any 
$\mu>0$ one of the solutions $\left\{C_{n}^{ \pm}\right\}$decays faster than the other; we denote this rapidly decaying solution by $\left\{C_{n}^{\prime}\right\}$. For example, if $\mu<1$, then $\left\{C_{n}^{\prime}\right\}=\left\{C_{n}^{+}\right\}$.

The elements $g_{n, k}$ satisfy $g_{n, k}=a C_{k}^{\prime} C_{n}^{\prime \prime}$, where $\left\{C_{n}^{\prime}\right\}$ is our rapidly decaying solution, $\left\{C_{n}^{\prime \prime}\right\}$ is another solution of the same system (a linear combination of the solutions $\left\{C_{n}^{ \pm}\right\}$), and $a$ is a constant. The elements of the matrix $\widetilde{\mathbf{Z}_{\gamma}}(i ; \mu)$ are equal to $(n+1 / 2)^{-\gamma} g_{n, k}(k+$ $1 / 2)^{-\gamma}$. Standard calculations show that $\widetilde{\mathbf{Z}_{\gamma}}(i ; \mu) \in \mathfrak{S}_{2}$ for any $\gamma>0$. Consequently, $\widetilde{\mathbf{Z}_{\gamma}}(i ; \mu) \in \mathfrak{S}_{1}$ for $\gamma>1 / 4$. Now, from (7.6) we deduce that $A \in \mathfrak{S}_{1}$ and $R \in \mathfrak{S}_{2}$, whence $\widetilde{\mathbf{Z}_{\gamma}}(\Lambda ; \mu) \in \mathfrak{S}_{1}$ for all $\Lambda \in \mathbb{C}_{+}$. Since $R \in \mathfrak{S}_{2}$, the operator-valued function $R^{*}(\mathcal{L}-\Lambda)^{-1} R$ has strong nontangential limits as $\Lambda \rightarrow \lambda$ for a.e. $\lambda \in \mathbb{R}$. Relation (7.5) shows that the same is true for the operator-valued function $\widetilde{\mathbf{Z}_{\gamma}}(\Lambda ; \mu)$.

For the function $\widetilde{\mathbf{Z}_{\gamma}^{\circ}}(\Lambda ; \mu)$ the proof is simpler, because formula (7.5) is not needed. The matrix $\left(\mathbf{J}^{\circ}(\mu)-\mu \Lambda\right)^{-1}$ can be expressed in terms of solutions of the homogeneous system

$$
\left(\mathbf{J}^{\circ}(\mu)-\mu \Lambda\right) X=0,
$$

which can be analyzed with the help of the Birkhoff-Adams theorem. By (7.2), the first two terms in the asymptotic expansions of the functions $p_{1}(n), p_{2}(n)$, obtained after reduction of (7.7) to the form (4.22), are the same as for the system with the matrix $\mathbf{J}(\Lambda ; \mu)$. Therefore, all calculations remain valid and lead to the same results.

3. Jumps of the functions $\widetilde{\mathbf{Z}_{\gamma}}(\Lambda ; \mu)$ and $\widetilde{\mathbf{Z}_{\gamma}^{\circ}}(\Lambda ; \mu)$ across the real axis. By Lemma 7.1, the jumps $\left[\widetilde{\mathbf{Z}_{\gamma}}\right](\lambda ; \mu)$ and $\left[\widetilde{\mathbf{Z}_{\gamma}^{\circ}}\right](\lambda ; \mu)$ exist for a.e. $\lambda \in \mathbb{R}$. Both jumps are bounded operators on $\ell^{2}$.

Lemma 7.2. Let $1 / 4<\gamma<1 / 2$. Then for any $\mu>0$ and for a.e. $\lambda \in(-\infty, 1 / 2)$ we have

$$
\operatorname{rank}\left[\widetilde{\mathbf{Z}_{\gamma}}\right](\lambda ; \mu)=\operatorname{rank}\left[\widetilde{\mathbf{Z}_{\gamma}^{\circ}}\right](\lambda ; \mu) .
$$

Proof. Consider the sequence of functions

$$
\phi_{n}(\Lambda)=(n+1 / 2)^{\gamma} \psi_{n}(\Lambda)(n+1 / 2)^{\gamma}, \quad \Lambda \notin[1 / 2, \infty)
$$

(recall that the functions $\psi_{n}$ were defined in (7.2)). If $\gamma<1 / 2$, then $\lim _{n \rightarrow \infty} \phi_{n}(\Lambda)=0$ for every $\Lambda$. Therefore, the operator

$$
\Phi(\Lambda)=\operatorname{diag}\left\{\phi_{n}(\Lambda)\right\}
$$

is compact in $\ell^{2}$. Obviously, the operator-valued function $\Phi(\Lambda)$ is analytic off the half-line $[1 / 2, \infty)$.

The Hilbert identity yields

$$
\widetilde{\mathbf{Z}_{\gamma}}(\Lambda ; \mu)-\widetilde{\mathbf{Z}_{\gamma}^{\circ}}(\Lambda ; \mu)=-\mu \widetilde{\mathbf{Z}_{\gamma}}(\Lambda ; \mu) \Phi(\Lambda) \widetilde{\mathbf{Z}_{\gamma}^{\circ}}(\Lambda ; \mu) .
$$

We subtract a similar identity for $\bar{\Lambda}$ and pass to the nontangential limits as $\Lambda \rightarrow \lambda$, where $\lambda \in(-\infty, 1 / 2)$. Since $\lim \Phi(\Lambda)=\lim \Phi(\bar{\Lambda})=\Phi(\lambda)$, we obtain

$$
\left.\left[\widetilde{\mathbf{Z}_{\gamma}^{\circ}}\right](\lambda ; \mu)-\left[\widetilde{\mathbf{Z}_{\gamma}}\right](\lambda ; \mu)=\mu\left(\widetilde{\mathbf{Z}_{\gamma}}\right](\lambda ; \mu) \Phi(\lambda) \widetilde{\mathbf{Z}_{\gamma}^{\circ}}(\lambda+i 0 ; \mu)+\widetilde{\mathbf{Z}_{\gamma}}(\lambda-i 0 ; \mu) \Phi(\lambda)\left[\widetilde{\mathbf{Z}_{\gamma}^{\circ}}\right](\lambda ; \mu)\right) .
$$

Consider the analytic (with respect to $\Lambda$ ) operator-valued functions

$$
\begin{array}{ll}
\mathcal{G}_{+}(\Lambda ; \mu)=I+\mu \Phi(\Lambda) \widetilde{\mathbf{Z}_{\gamma}^{\circ}}(\Lambda ; \mu), & \Lambda \in \mathbb{C}_{+} ; \\
\mathcal{G}_{-}(\Lambda ; \mu)=I-\mu \widetilde{\mathbf{Z}_{\gamma}}(\Lambda ; \mu) \Phi(\Lambda), & \Lambda \in \mathbb{C}_{-} .
\end{array}
$$


By Lemma 7.1, both functions have nontangential limits as $\Lambda \rightarrow \lambda$ for a.e. $\lambda \in(-\infty, 1 / 2)$. Moreover, the operators $\mathcal{G}_{ \pm}(\lambda \pm i 0 ; \mu)-I$ are compact in $\ell^{2}$. Identity (7.8) implies that

$$
\mathcal{G}_{-}(\lambda-i 0 ; \mu)\left[\widetilde{\mathbf{Z}_{\gamma}^{\circ}}\right](\lambda ; \mu)=\left[\widetilde{\mathbf{Z}_{\gamma}}\right](\lambda ; \mu) \mathcal{G}_{+}(\lambda+i 0 ; \mu)
$$

for a.e. $\lambda$. Next, it can be shown that $\operatorname{Ker} \mathcal{G}_{ \pm}(\Lambda ; \mu)=\{0\}$ for any $\Lambda \in \mathbb{C}_{ \pm}$. This was proved in [14, Subsection 7.3] in the case where $\Gamma=\mathbb{R}$. The proof for the general situation stays the same. By a result of Naboko [10, Theorem 3], the properties of the functions $\mathcal{G}_{ \pm}$imply that $\operatorname{Ker} \mathcal{G}_{ \pm}(\lambda \pm i 0 ; \mu)=\{0\}$ for a.e. $\lambda \in(-\infty, 1 / 2)$. Now, the statement of the lemma follows from (7.9).

\section{§8. Absolutely continuous spectrum: End of the proof of Theorem 3.6}

As was already noted in Subsection 6.2, it suffices to prove formula (3.6) for the multiplicity function $\mathfrak{m}_{\text {a.c. }}$.

1. The case of $\lambda<1 / 2$. Along with the operator $\widetilde{\mathbf{G}}_{\gamma}$ in $\ell^{2}$ (see (7.4)), we consider a similar operator $\mathbf{G}_{\gamma}$ acting in $\mathfrak{H}=\ell^{2}\left(\mathbb{N}_{0} ; L^{2}(\Gamma)\right)$ :

$$
\mathbf{G}_{\gamma}:\left\{u_{n}(x)\right\} \mapsto\left\{(n+1 / 2)^{-\gamma} u_{n}(x)\right\} .
$$

The operator $\mathbf{G}_{\gamma}$ is selfadjoint but, unlike $\widetilde{\mathbf{G}}_{\gamma}$, is not compact. Note that

$$
\mathbf{G}_{\gamma} \mathbf{T}(\Lambda)=\mathbf{T}(\Lambda) \widetilde{\mathbf{G}}_{\gamma}, \quad \mathbf{T}(\bar{\Lambda})^{*} \mathbf{G}_{\gamma}=\widetilde{\mathbf{G}}_{\gamma} \mathbf{T}(\bar{\Lambda})^{*} .
$$

For any $\alpha \geq 0$ and $\Lambda \neq \bar{\Lambda}$, we put

$$
\mathbf{Z}_{\gamma}(\Lambda ; \alpha)=\mathbf{G}_{\gamma}\left(\mathbf{A}_{\alpha}-\Lambda\right)^{-1} \mathbf{G}_{\gamma} .
$$

Then, by (8.1) and Theorem 6.1, we have

$$
\mathbf{Z}_{\gamma}(\Lambda ; \alpha)=\mathbf{Z}_{\gamma}(\Lambda ; 0)+\mathbf{T}(\Lambda)\left(\mu \widetilde{\mathbf{Z}_{\gamma}}(\Lambda ; \mu)-\widetilde{\mathbf{G}}_{\gamma}(d(o) \mathbf{Y}(\Lambda))^{-1} \widetilde{\mathbf{G}}_{\gamma}\right) \mathbf{T}(\bar{\Lambda})^{*},
$$

where, as usual, $\mu=\mu(\alpha)=d(o)(\alpha \sqrt{2})^{-1}$. We calculate the jumps of the operator-valued functions in (8.2) at the point $\lambda<1 / 2$. Since the operator-valued functions $\mathbf{Z}_{\gamma}(\Lambda ; 0)$, $\mathbf{Y}(\Lambda)^{-1}, \mathbf{T}(\Lambda)$, and $\mathbf{T}(\bar{\Lambda})^{*}$ are analytic in a neighborhood of $\lambda$, for a.e. $\lambda$ we have

$$
\left[\mathbf{Z}_{\gamma}\right](\lambda ; 0)=0, \quad\left[\mathbf{Y}^{-1}\right](\lambda)=0 .
$$

Thus,

$$
\left[\mathbf{Z}_{\gamma}\right](\lambda ; \alpha)=\mu \mathbf{T}(\lambda)\left[\widetilde{\mathbf{Z}_{\gamma}}\right](\lambda ; \mu) \mathbf{T}(\lambda)^{*}, \quad \mu=\mu(\alpha) .
$$

In particular, this computation shows that the operator-valued function on the left-hand side in (8.2) has a jump. Combining this with Lemma 7.2, we obtain

$$
\operatorname{rank}\left[\mathbf{Z}_{\gamma}\right](\lambda ; \alpha)=\operatorname{rank}\left[\widetilde{\mathbf{Z}_{\gamma}^{\circ}}\right](\lambda ; \mu(\alpha)) .
$$

Applying Theorem 6.2, we see that $\mathfrak{m}_{\text {a.c. }}\left(\lambda ; \mathbf{A}_{\alpha}\right)=\mathfrak{m}_{\text {a.c. }}\left(\lambda ; \mathbf{J}^{\circ}(\mu(\alpha))\right)$ for a.e. $\lambda<1 / 2$. For such $\lambda$ this coincides with (3.6).

2. The case of $\lambda>1 / 2$. The proof can be extended to the values $\lambda>1 / 2$ with the help of a simple technical trick based on passage to the subspace $\mathfrak{H}^{\prime}=\mathfrak{H}_{m}^{\prime}=\ell^{2}\left(\mathbb{N}_{m} ; L^{2}(\Gamma)\right)$ for sufficiently large $m$. Here $\mathbb{N}_{m}=\{m, m+1, \ldots\}$ for any $m \in \mathbb{N}$. The subspace $\mathfrak{H}^{\prime}$ is not invariant with respect to $\mathbf{A}_{\alpha}$. However, this subspace is invariant with respect to an operator $\mathbf{A}_{\alpha}^{\prime \prime}$ obtained from $\mathbf{A}_{\alpha}$ by perturbation of its resolvent by an appropriate operator of finite rank. For the operator $\mathbf{A}_{\alpha}^{\prime \prime}$, the method developed in Subsection 8.1 works if $\lambda<m+1 / 2$. At the same time, returning to the operator $\mathbf{A}_{\alpha}$ does not change the absolutely continuous spectrum, because complete wave operators exist for the pair $\mathbf{A}_{\alpha}, \mathbf{A}_{\alpha}^{\prime \prime}$ (see, e.g., [16, Theorem 6.5.1]).

The same trick will be used in Subsection 9.2 for the proof of statement 2) of Theorem 3.2, as well as in Subsection 9.3, where another proof of statement 1) of Theorem 3.1 
will be outlined. There, the application of the trick is simpler, because for $\alpha \sqrt{2}<d(o)$ we can use quadratic forms.

So, let $\lambda>1 / 2$. We fix a number $m>\lambda-1 / 2$ and consider the Hilbert space $\mathfrak{H}^{\prime}=\mathfrak{H}_{m}^{\prime}=\ell^{2}\left(\mathbb{N}_{m} ; L^{2}(\Gamma)\right)$. We consider a family of operators $\mathbf{A}_{\alpha}^{\prime}$ in $\mathfrak{H}^{\prime}$. The definition of this family is similar to that of the operators $\mathbf{A}_{\alpha}$ in $\S 2$. The only difference is that now we have $n \in \mathbb{N}_{m}$, and condition (2.7) for $n=m$ is replaced by

$$
\sqrt{2}\left[u_{m}^{\prime}\right](o)=\alpha \sqrt{m+1} u_{m+1}(o) .
$$

The operators $\mathbf{A}_{\alpha}^{\prime}$ are selfadjoint in $\mathfrak{H}^{\prime}$, which can be proved as in Theorem 2.1, and the absolutely continuous spectrum of $\mathbf{A}_{\alpha}^{\prime}$ can be investigated by the same method. The definitions of the corresponding operators $\left(\mathbf{J}^{\circ}\right)^{\prime}(\mu), \mathbf{J}^{\prime}(\Lambda ; \mu), \mathbf{Y}^{\prime}(\Lambda)$, and $\mathbf{T}^{\prime}(\Lambda)$ differ from (3.3), (4.20), (6.1), and (6.2) only by the fact that now $n \in \mathbb{N}_{m}$. The operator $\left(\mathbf{J}^{\circ}\right)^{\prime}(\mu)$ is obtained from $\mathbf{J}^{\circ}(\mu)$ by passage to a subspace of finite codimension. This does not affect the absolute continuous spectrum. It is important that

$$
\sigma\left(\mathbf{A}_{0}^{\prime}\right) \subset[m+1 / 2, \infty) .
$$

Therefore, for the operator-valued functions $\mathbf{J}^{\prime}(\Lambda ; \mu), \mathbf{Y}^{\prime}(\Lambda)$, and $\mathbf{T}^{\prime}(\Lambda)$, the domain of analyticity is wider; now this domain is $\mathbb{C} \backslash[m+1 / 2, \infty)$.

The result of Theorem 6.1 carries over to the new situation with only one change: all operators occurring in (6.4) should be marked with a prime. The analog of Lemma 7.2 remains valid, with $\lambda \in(-\infty, m+1 / 2)$. Arguing as in Subsection 8.1, we show that, for a.e. $\lambda<m+1 / 2$,

$$
\mathfrak{m}_{\text {a.c. }}\left(\lambda ; \mathbf{A}_{\alpha}^{\prime}\right)=\mathfrak{m}_{\text {a.c. }}\left(\lambda ;\left(\mathbf{J}^{\circ}\right)^{\prime}(\mu)\right)=\mathfrak{m}_{\text {a.c. }}\left(\lambda ; \mathbf{J}^{\circ}(\mu)\right), \quad \mu=\mu(\alpha) .
$$

Now, we consider the following operator $\mathbf{A}_{\alpha}^{\prime \prime}$ in the initial space $\mathfrak{H}$ :

$$
\mathbf{A}_{\alpha}^{\prime \prime}=\left(\sum_{n<m}^{\oplus}(-\Delta+(n+1 / 2))\right) \oplus \mathbf{A}_{\alpha}^{\prime} .
$$

Clearly,

$$
\mathfrak{m}_{\text {a.c. }}\left(\lambda ; \mathbf{A}_{\alpha}^{\prime \prime}\right)=\sum_{n<m} \mathfrak{m}_{\text {a.c. }}(\lambda ;-\Delta+(n+1 / 2))+\mathfrak{m}_{\text {a.c. }}\left(\lambda ; \mathbf{A}_{\alpha}^{\prime}\right) .
$$

Observe that for $\lambda<m+1 / 2$ the first sum on the right coincides with $\mathfrak{m}_{\text {a.c. }}\left(\lambda ; \mathbf{A}_{0}\right)$.

The operator (8.3) can be rewritten as $\mathbf{A}_{\alpha}^{\prime \prime}=\mathcal{A} \backslash \mathcal{D}_{\alpha}^{\prime \prime}$, where the set $\mathcal{D}_{\alpha}^{\prime \prime}$ differs from the set $\mathcal{D}_{\alpha}$ described in Subsection 2.2 only by conditions on the functions $u_{n}$ with $n \leq m$ at the point $o$. Now these conditions look like this:

$$
\left[u_{n}^{\prime}\right](o)=0, \quad n<m ; \quad \sqrt{2}\left[u_{m}^{\prime}\right](o)=\alpha \sqrt{m+1} u_{m+1}(o) .
$$

This implies that the difference of the resolvents of $\mathbf{A}_{\alpha}$ and $\mathbf{A}_{\alpha}^{\prime \prime}$ is a finite rank operator. Thus, for both operators the absolutely continuous spectra and the multiplicity functions are the same. Since, by (2.8),

$$
\mathfrak{m}_{\text {a.c. }}\left(\lambda ; \mathbf{A}_{0}\right)=\sum_{n<m} \mathfrak{m}_{\text {a.c. }}(-\Delta+(n+1 / 2))
$$

for a.e. $\lambda<m+1 / 2$, identity (8.4) implies (3.6).

\section{§9. The operator $\mathbf{A}_{\alpha}$ for small $\alpha$ : Application of quadratic forms}

For small $\alpha$, i.e., for $\alpha \sqrt{2}<d(o)$, we can apply quadratic forms to study the operator $\mathbf{A}_{\alpha}$. This will allow us to describe the point spectrum below the threshold point $\lambda=1 / 2$ in detail; also, we give a simpler proof of statement 1) of Theorem 3.1. This approach also works in the extreme case where $\alpha \sqrt{2}=d(o)$. However, the results obtained in this case are less complete. 
1. The quadratic form of the operator $\mathbf{A}_{\alpha}$. Formally, the operator $\mathbf{A}_{\alpha}$ corresponds to the quadratic form

$$
\mathbf{a}_{\alpha}[U]=\mathbf{a}_{0}[U]+\alpha \mathbf{b}[U]
$$

where

$$
\mathbf{a}_{0}[U]=\int_{\Gamma \times \mathbb{R}}\left(\left|U_{x}^{\prime}\right|^{2}+\frac{1}{2}\left(\left|U_{q}^{\prime}\right|^{2}+q^{2}|U|^{2}\right)\right) d x d q, \quad \mathbf{b}[U]=\int_{\mathbb{R}} q|U(o, q)|^{2} d q .
$$

The quadratic form $\mathbf{a}_{0}$ is positive definite and closed on the natural domain Dom $\mathbf{a}_{0}$ defined by the requirement that the expression $\mathbf{a}_{0}[U]$ be finite. In the representation (2.4), $\mathbf{a}_{0}$ takes the form

$$
\mathbf{a}_{0}[U]=\sum_{n \in \mathbb{N}_{0}} a_{n}\left[u_{n}\right]:=\sum_{n \in \mathbb{N}_{0}} \int_{\Gamma}\left(\left|u_{n}^{\prime}\right|^{2}+(n+1 / 2)\left|u_{n}\right|^{2}\right) d x .
$$

For the quadratic form $\mathbf{b}$, we use (2.3) to write

$$
\mathbf{b}[U]=\sum_{n \in \mathbb{N}} \sqrt{2 n} \operatorname{Re}\left(u_{n}(o) \overline{u_{n-1}(o)}\right) .
$$

The next estimate can be obtained with the help of the standard technique of the calculus of variations; we omit the proof.

Lemma 9.1. Suppose $h \in H^{1}(0, l), l<\infty$, and $\gamma>0$. Then

$$
|h(0)|^{2} \leq \gamma^{-1} \operatorname{coth}(\gamma l) \int_{0}^{l}\left(\left|h^{\prime}\right|^{2}+\gamma^{2}|h|^{2}\right) d x .
$$

Equality in (9.2) is attained on the one-dimensional space generated by the function $h_{\gamma}(x)=\cosh (\gamma(l-x))$.

Remark 9.2. For $l=\infty$, relation (9.2) turns into

$$
|h(0)|^{2} \leq \gamma^{-1} \int_{0}^{\infty}\left(\left|h^{\prime}\right|^{2}+\gamma^{2}|h|^{2}\right) d x .
$$

The extremal subspace for this inequality is generated by the function $e^{-\gamma x}$.

Corollary 9.3. Suppose $\Gamma \in \mathfrak{G}, o \in \Gamma$, and $\gamma>0$. Then for any function $w \in H^{1}(\Gamma)$ we have

$$
|w(o)|^{2} \leq \frac{\operatorname{coth}(\gamma \varepsilon(\Gamma, o))}{\gamma d(o)} \int_{\Gamma}\left(\left|w^{\prime}\right|^{2}+\gamma^{2}|w|^{2}\right) d x, \quad \varepsilon(\Gamma, o)=\min \{|e|: e \subset \mathcal{S}(o)\} .
$$

For the proof, it suffices to replace the integral over $\Gamma$ by the integral over $\mathcal{S}(o)$ and to apply (9.2) for every edge $e \in \mathcal{S}(o)$.

Recall that the $\mathbf{a}_{0}$-bound of the quadratic form $\mathbf{b}$ is the greatest lower bound of the constants $C$ such that

$$
|\mathbf{b}[U]| \leq C \mathbf{a}_{0}[U]+P\|U\|_{\mathfrak{H}}^{2}, \quad U \in \operatorname{Dom} \mathbf{a}_{0},
$$

for some nonnegative $P=P(C)$.

Lemma 9.4. There exists a number $R \geq 0$ such that

$$
\frac{d(o)}{\sqrt{2}}|\mathbf{b}[U]| \leq \mathbf{a}_{0}[U]+R\|U\|_{\mathfrak{H}}^{2}, \quad U \in \operatorname{Dom} \mathbf{a}_{0} .
$$

Here, the factor 1 before $\mathbf{a}_{0}[U]$ is best possible. In particular, the $\mathbf{a}_{0}$-bound of the quadratic form $\mathbf{b}$ is equal to

$$
Q\left(\mathbf{b} ; \mathbf{a}_{0}\right)=\sqrt{2} / d(o) .
$$


The claim of this lemma is slightly stronger than merely a statement about the value of the $\mathbf{a}_{0}$-bound of $\mathbf{b}$ : this claim also shows that the lower bound of the constants $C$ in (9.4) is attained.

Proof of the lemma. For $U \in \operatorname{Dom} \mathbf{a}_{0}$, from (9.1) we deduce that

$$
\begin{aligned}
\sqrt{2}|\mathbf{b}[U]| & \leq 2 \sum_{n=1}^{\infty} \sqrt{n}\left|u_{n}(o)\right|\left|u_{n-1}(o)\right| \leq \sum_{n=1}^{\infty} \sqrt{n}\left(\left|u_{n}(o)\right|^{2}+\left|u_{n-1}(o)\right|^{2}\right) \\
& =\sum_{n=0}^{\infty}(\sqrt{n}+\sqrt{n+1})\left|u_{n}(o)\right|^{2} \leq \sum_{n=0}^{\infty} \sqrt{2(2 n+1)}\left|u_{n}(o)\right|^{2}
\end{aligned}
$$

Let $R$ be a constant; we shall specify it later. For brevity, we denote $\varepsilon=\varepsilon(\Gamma, o)$. By $(9.3)$, we have

$$
\begin{aligned}
d(o) \sum_{n=0}^{\infty} \sqrt{2(2 n+1)}\left|u_{n}(o)\right|^{2} \leq 2 \sum_{n=0}^{\infty} & \sqrt{\frac{n+1 / 2}{n+1 / 2+R}} \operatorname{coth}(\varepsilon \sqrt{n+1 / 2+R}) \\
& \times \int_{\Gamma}\left(\left|u_{n}^{\prime}\right|^{2}+(n+1 / 2+R)\left|u_{n}\right|^{2}\right) d x .
\end{aligned}
$$

The coefficient in front of the integral is a product in which, as $n \rightarrow \infty$, the first factor tends to one as a power and the second factor tends to one exponentially. Therefore, for sufficiently large $R$ this coefficient is smaller than 1 for all $n$. This shows that inequalities (9.6) and (9.7) imply (9.5).

By (9.5), for $\alpha \sqrt{2} \leq d(o)$ the quadratic form $\mathbf{a}_{\alpha}$ is lower semibounded and closed on the domain Dom $\mathbf{a}_{0}$. By statement 1) in Theorem 3.3, the operator $\mathbf{A}_{\alpha}$ is not semibounded for $\alpha \sqrt{2}>d(o)$. Thus, the coefficient of $\mathbf{a}_{0}[U]$ in (9.5) cannot be reduced.

Remark 9.5. In the case of a star graph $\Gamma$ with center vertex $o$ and with Dirichlet condition on $\partial \Gamma$, inequality (9.5) is fulfilled with $R=0$ (see [13]). This is no longer true for the Neumann condition. For a general graph $\Gamma \in \mathfrak{G}$, inequality (9.5) with $R=0$ may fail even for the Dirichlet condition, which can be shown by simple examples.

2. Proof of statement 2) in Theorem 3.2. Here we prove that the negative spectrum of the operator $\mathbf{A}_{d(o) / \sqrt{2}}$ is discrete. First, by (9.5) we have

$$
\mathbf{a}_{d(o) / \sqrt{2}}[U] \geq-R\|U\|_{\mathfrak{H}}^{2}, \quad U \in \operatorname{Dom} \mathbf{a}_{0},
$$

so that the operator $\mathbf{A}_{d(o) / \sqrt{2}}$ is lower semibounded. To prove the discreteness of the negative spectrum, we fix $m \in \mathbb{N}$ and consider the subspace $D_{m} \subset$ Dom $\mathbf{a}_{0}$ defined by the conditions $u_{n}(o)=0, n=0, \ldots, m$. The codimension of this subspace is finite. On the elements of $D_{m}$, inequality (9.6) takes the form

$$
\sqrt{2}|\mathbf{b}[U]| \leq \sum_{n>m} \sqrt{2(2 n+1)}\left|u_{n}(o)\right|^{2} .
$$

Therefore, on the right-hand side in (9.7) it suffices to take the sum over $n>m$. Let $\psi$ be the function defined in the proof of Lemma 9.4. For an arbitrarily small $R=\varepsilon>0$, we can find $m=m(R)$ such that $\psi(n+1 / 2+R) \leq 1$ for all $n>m$. It follows that, along with inequality (9.5), for every $\varepsilon>0$ we have

$$
\mathbf{a}_{d(o) / \sqrt{2}}[U] \geq-\varepsilon\|U\|_{\mathfrak{H}}^{2}, \quad U \in D_{m}, \quad m=m(\varepsilon) .
$$

This completes the proof. 
3. On an alternative proof of statement 1) in Theorem 3.1. For $\alpha \sqrt{2}<d(o)$, the absolutely continuous spectrum of the operator $\mathbf{A}_{\alpha}$ can be analyzed by purely variational methods without reference to Theorem 3.6.

As in Subsection 8.2, let $\mathfrak{H}^{\prime}=\mathfrak{H}_{m}^{\prime}=\ell^{2}\left(\mathbb{N}_{m} ; L^{2}(\Gamma)\right)$, where $m$ is an integer and $\mathbb{N}_{m}=\{m, m+1, \ldots\}$. In $\mathfrak{H}^{\prime}$ consider the quadratic form

$$
\begin{aligned}
\mathbf{a}_{\alpha}^{\prime}[U] & =\mathbf{a}_{0}^{\prime}[U]+\alpha \mathbf{b}^{\prime}[U] \\
& :=\sum_{n \geq m} \int_{\Gamma}\left(\left|u_{n}^{\prime}\right|^{2}+(n+1 / 2)\left|u_{n}\right|^{2}\right) d x+\alpha \sum_{n>m} \sqrt{2 n} \operatorname{Re}\left(u_{n}(o) \overline{u_{n-1}(o)}\right) .
\end{aligned}
$$

Obviously,

$$
\mathbf{a}_{0}^{\prime}[U] \geq(m+1 / 2)\|U\|_{\mathfrak{H}^{\prime}}^{2} .
$$

It is also clear that the quadratic form $\mathbf{a}_{0}^{\prime}$ is positive definite and closed on the natural domain Dom $\mathbf{a}_{0}^{\prime}$. Lemma 9.4 remains valid for the quadratic forms $\mathbf{a}_{0}^{\prime}$ and $\mathbf{b}^{\prime}$ and shows that for $\alpha \sqrt{2}<d(o)$ the quadratic form $\mathbf{a}_{\alpha}^{\prime}$ is positive definite and closed on Dom $\mathbf{a}_{0}^{\prime}$. It is easy to realize that $\mathbf{a}_{\alpha}^{\prime}$ is the quadratic form of the operator $\mathbf{A}_{\alpha}^{\prime}$ introduced in Subsection 8.2. Recall that this operator depends on the parameter $m$, which is not indicated in the notation for brevity.

Combining the identity

$$
\mathbf{a}_{\alpha}^{\prime}[U]=\frac{\alpha \sqrt{2}}{d(o)}\left(\mathbf{a}_{0}^{\prime}[U]+\frac{d(o)}{\sqrt{2}} \mathbf{b}^{\prime}[U]\right)+\left(1-\frac{\alpha \sqrt{2}}{d(o)}\right) \mathbf{a}_{0}^{\prime}[U]
$$

with inequalities (9.5) and (9.8), we obtain

$$
\mathbf{a}_{\alpha}^{\prime}[U] \geq\left(\left(1-\frac{\alpha \sqrt{2}}{d(o)}\right)(m+1 / 2)-\frac{\alpha \sqrt{2}}{d(o)} R\right)\|U\|_{\mathfrak{H}^{\prime}}^{2}, \quad U \in \operatorname{Dom} \mathbf{a}_{0}^{\prime} .
$$

This shows that, for $\alpha \sqrt{2}<d(o)$ and sufficiently large $m$, the spectrum of the operator $\mathbf{A}_{\alpha}^{\prime}$ is a subset of the half-line $(\lambda(m), \infty)$, where $\lambda(m) \rightarrow \infty$ as $m \rightarrow \infty$. Therefore, the spectrum of the operator $\mathbf{A}_{\alpha}^{\prime \prime}$ (see (8.3)) below the threshold point $\lambda(m)$ is the same as the spectrum of the orthogonal sum of the operators $-\Delta+(n+1 / 2)$ over all $n<m$. Since the absolutely continuous spectra of the operators $\mathbf{A}_{\alpha}$ and $\mathbf{A}_{\alpha}^{\prime \prime}$ and their multiplicities do coincide, the relation $\mathfrak{m}_{\text {a.c. }}\left(\lambda ; \mathbf{A}_{\alpha}\right)=\mathfrak{m}_{\text {a.c. }}\left(\lambda ; \mathbf{A}_{\alpha}^{\prime \prime}\right)$ is fulfilled for all $\lambda<\lambda(m)$. Letting $m \rightarrow \infty$, we obtain the result.

§10. The operator $\mathbf{A}_{\alpha}$ for small $\alpha$ : The spectrum on the half-Line $\lambda<1 / 2$, QUANTITATIVE RESULTS

If the graph $\Gamma$ is compact, then the spectrum of $\mathbf{A}_{0}$ is discrete (see Theorem 2.2). Statement 1) in Theorem 3.1 shows that for $\alpha \sqrt{2}<d(o)$ the spectrum of $\mathbf{A}_{\alpha}$ is also discrete. If the graph is noncompact, then $\sigma\left(\mathbf{A}_{0}\right)=[1 / 2, \infty)$, and the property of the relative boundedness of the perturbation is not sufficient to make informative conclusions about the spectrum of $\mathbf{A}_{\alpha}$ below the threshold point 1/2. Nevertheless, Statements 2) and 3) of Theorem 3.1 describe this part of the spectrum in detail. These statements are proved in the present section.

1. Nonemptiness of the spectrum on $(-\infty, 1 / 2)$. It suffices to find an element $U \in \operatorname{Dom} \mathbf{a}_{0}$ such that $2 \mathbf{a}_{\alpha}[U]<\|U\|_{\mathfrak{H}}^{2}$. Let $U \sim\left\{u_{n}\right\}$, where only the functions $u_{0}$ and $u_{1}$ are not equal to zero. We take them to be real-valued and such that $u_{0}(o):=-C<0$, $u_{1}(o)=1$. Then

$$
\mathbf{a}_{\alpha}[U]-\|U\|_{\mathfrak{H}}^{2} / 2=\int_{\Gamma}\left(\left|u_{0}^{\prime}\right|^{2}+\left|u_{1}^{\prime}\right|^{2}+\left|u_{1}\right|^{2}\right) d x-C \alpha \sqrt{2}
$$


First, assume that the graph $\Gamma$ is noncompact and set $E=\max \{\rho(o, v): v \in \mathcal{V}(\Gamma)\}$. If $x \in \Gamma$ and $\rho(o, x)>E$, then the point $x$ lies on an edge $e$ of infinite length. If $\epsilon>0$ and $\rho(o, x)>E \max (1, \epsilon)$, then there exists a unique point $x^{\epsilon} \in \Gamma$ belonging to the same edge and such that $\rho\left(o, x^{\epsilon}\right)=\epsilon \rho(o, x)$.

We choose the function $u_{0}$ in such a way that $u_{0}(x)=1$ for $\rho(o, x) \leq E$. Then for $\epsilon<1$ the function

$$
u_{0}^{\epsilon}(x)= \begin{cases}1 & \text { if } \rho(o, x) \leq E \epsilon^{-1} \\ u_{0}\left(x^{\epsilon}\right) & \text { if } \rho(o, x)>E \epsilon^{-1}\end{cases}
$$

is well defined. For the element $U^{\epsilon}=\left\{u_{0}^{\epsilon}, u_{1}, 0,0, \ldots\right\}$ we have

$$
\mathbf{a}_{\alpha}\left[U^{\epsilon}\right]-\left\|U^{\epsilon}\right\|_{\mathfrak{H}}^{2} / 2=\int_{\Gamma}\left(\epsilon\left|u_{0}^{\prime}\right|^{2}+\left|u_{1}^{\prime}\right|^{2}+\left|u_{1}\right|^{2}\right) d x-C \alpha \sqrt{2} .
$$

Choosing a sufficiently small $\epsilon$ and a sufficiently large $C$, we can ensure that the inequality $2 \mathbf{a}_{\alpha}\left[U^{\epsilon}\right]<\left\|U^{\epsilon}\right\|_{\mathfrak{H}}^{2}$ be fulfilled for any $\alpha>0$. Consequently, the spectrum on the half-line $\lambda<1 / 2$ is nonempty.

If the graph is compact, then the proof simplifies, because then $\lambda=1 / 2$ is an eigenvalue of $\mathbf{A}_{0}$ with the eigenfunction $U \sim\left\{u_{0}, 0,0, \ldots\right\}, u_{0}(x) \equiv 1$. It is easy to find an element $U^{\prime}$ in a neighborhood of $U$ such that $2 \mathbf{a}_{\alpha}\left[U^{\prime}\right]<\left\|U^{\prime}\right\|_{\mathfrak{H}}^{2}$. The reader can easily reconstruct the argument.

2. The finiteness of the spectrum on the half-line $\lambda<1 / 2$. The proof is based on Theorem 10.1, which relates the spectrum of $\mathbf{A}_{\alpha}$ below the threshold point $1 / 2$ to the spectrum of some Jacobi matrix. This theorem can be viewed as a special case of the general Birman-Schwinger principle.

We fix a number $\eta \in(0,1 / 2)$ and consider a zero-diagonal Jacobi matrix $\mathbf{J}_{\eta}$ :

$$
\mathbf{J}_{\eta}=\mathbf{J}\left(0,\left\{\beta_{n}(\eta)\right\}\right), \quad \beta_{n}(\eta)=\frac{d(o) \sqrt{n}}{2 \sqrt{r(n+\eta ; o) r(n-1+\eta ; o)}} .
$$

Recall that the numbers $r(\zeta ; o)$ were defined in (4.1). Along with $\mathbf{J}_{\eta}$, we need the matrix $\mathbf{J}_{\eta}^{\prime}$ obtained from $\mathbf{J}_{\eta}$ by deleting the upper row and the left column: $\mathbf{J}_{\eta}^{\prime}=\mathbf{J}\left(0,\left\{\beta_{n+1}(\eta)\right\}\right)$. Unlike $\mathbf{J}_{\eta}$, the matrix $\mathbf{J}_{\eta}^{\prime}$ itself is well defined also for $\eta=0$. The matrix $\mathbf{J}_{0}$ itself is not defined since the value $r(0 ; o)$ is meaningless.

By (4.8), the sequence $\left\{\beta_{n}(\eta)\right\}$ has the following asymptotics in $n$ (for fixed $\eta$ ):

$$
\beta_{n}(\eta) \sim 1 / 2+\frac{1-2 \eta}{8 n} .
$$

It is well known that off the interval $[-1,1]$ the spectrum of the Jacobi matrices $\mathbf{J}\left(0,\left\{\beta_{n}\right\}\right)$ such that $\beta_{n} \rightarrow 1 / 2$ is discrete. Since the matrix has zero diagonal, its spectrum is symmetric with respect to the point $\lambda=0$. Thus, in particular,

$$
N_{+}\left(s ; \mathbf{J}_{\eta}\right)=N_{-}\left(-s ; \mathbf{J}_{\eta}\right), \quad s>1 .
$$

Theorem 10.1. If $\alpha \sqrt{2}<d(o)$ and $0<\eta<1 / 2$, then

$$
N_{-}\left(1 / 2-\eta ; \mathbf{A}_{\alpha}\right)=N_{+}\left(\mu(\alpha) ; \mathbf{J}_{\eta}\right), \quad \mu(\alpha)=\frac{d(o)}{\alpha \sqrt{2}} .
$$

Proof. The variational principle yields

$$
N_{-}\left(1 / 2-\eta ; \mathbf{A}_{\alpha}\right)=\max _{\mathcal{F} \in \mathfrak{F}(\eta)} \operatorname{dim} \mathcal{F},
$$

where $\mathfrak{F}(\eta)$ is the set of linear subspaces $\mathcal{F} \subset$ Dom $\mathbf{a}_{0}$ such that

$$
\mathbf{a}_{\alpha}[U]<(1 / 2-\eta)\|U\|_{\mathfrak{H}}^{2} \quad \text { for all } U \in \mathcal{F}, \quad U \neq 0 .
$$


We put

$$
\|U\|_{\eta}^{2}=\sum_{n \in \mathbb{N}_{0}} \int_{\Gamma}\left(\left|u_{n}^{\prime}\right|^{2}+(n+\eta)\left|u_{n}\right|^{2}\right) d x, \quad U \sim\left\{u_{n}\right\} \in \operatorname{Dom} \mathbf{a}_{0}
$$

Let $\mathcal{D}_{\eta}$ denote the set Dom $\mathbf{a}_{0}$ viewed as a Hilbert space with respect to the metric $\|U\|_{\eta}$. Obviously, the metrics $\|U\|_{\eta}$ are mutually equivalent for all $\eta>0$. By Lemma 9.4, the quadratic form $\mathbf{b}[U]$ determines a bounded operator $\mathbf{T}_{\eta}$ in $\mathcal{D}_{\eta}$. Inequality (10.5) can be rewritten as

$$
\alpha \mathbf{b}[U]<-\|U\|_{\eta}^{2}
$$

Comparing (10.5) and (10.6), we see that

$$
N_{-}\left(1 / 2-\eta ; \mathbf{A}_{\alpha}\right)=N_{-}\left(-\alpha^{-1} ; \mathbf{T}_{\eta}\right)
$$

Consider the subspace $\mathcal{M}_{\eta} \subset \mathcal{D}_{\eta}$ formed by the elements

$$
U \sim\left\{C_{n} \frac{g(n+\eta ; x, o)}{\sqrt{r(n+\eta ; o)}}\right\}, \quad X:=\left\{C_{n}\right\} \in \ell^{2} .
$$

By (4.4), we have $\|U\|_{\eta}=\|X\|_{\ell^{2}}$.

In accordance with (4.3), the projection from $\mathcal{D}_{\eta}$ onto $\mathcal{M}_{\eta}$ is given by

$$
\left\{u_{n}\right\} \mapsto\left\{u_{n}(o) \frac{g(n+\eta ; ., o)}{\sqrt{r(n+\eta ; o)}}\right\} .
$$

Therefore, the orthogonal complement (in $\mathcal{D}_{\eta}$ ) to the subspace $\mathcal{M}_{\eta}$ is a subset of the kernel of the operator $\mathbf{T}_{\eta}$. The quantity $N_{-}\left(\alpha^{-1} ; \mathbf{T}_{\eta}\right)$ does not change if we replace the operator $\mathbf{T}_{\eta}$ by its restriction to $\mathcal{M}_{\eta}$, or in other words, if we replace the element $U$ in (10.6) by its projection to $\mathcal{M}_{\eta}$. This replacement leads to the inequality

$$
\sum_{n \in \mathbb{N}} \frac{\sqrt{2 n} \operatorname{Re}\left(C_{n} \overline{C_{n-1}}\right)}{\sqrt{r(n+\eta ; o) r(n-1+\eta ; o)}}<-\alpha^{-1} \sum_{n \in \mathbb{N}_{0}}\left|C_{n}\right|^{2},
$$

or equivalently, $\left(\mathbf{J}_{\eta} X, X\right)_{\ell^{2}}<-\mu(\alpha)\|X\|_{\ell^{2}}^{2}$. Using the variational principle for the spectrum of the matrix $\mathbf{J}_{\eta}$ and taking (10.3) into account, we obtain (10.4).

Identity (10.4) can be viewed as a realization of the general Birman-Schwinger principle for the family of operators $\mathbf{A}_{\alpha}$. However, this identity does not follow directly from the principle, i.e., in our case, from (10.7). The new moment here is the "separation of the subspace ker $\mathbf{T}$ ", i.e., passage to a variational problem in the subspace $\mathcal{M}_{\eta}$.

Theorem 10.1 was proved in [15], where the problem was considered for $\Gamma=\mathbb{R}$. The above presentation emphasizes the relationship with the Birman-Schwinger principle in its classical form.

By (10.4), we have $\left|N_{-}\left(1 / 2-\eta ; \mathbf{A}_{\alpha}\right)-N_{+}\left(\mu(\alpha) ; \mathbf{J}_{\eta}^{\prime}\right)\right| \leq 1$. Letting $\eta \rightarrow 0$, we obtain

$$
\left|N_{-}\left(1 / 2 ; \mathbf{A}_{\alpha}\right)-N_{+}\left(\mu(\alpha) ; \mathbf{J}_{0}^{\prime}\right)\right| \leq 1,
$$

which implies statement 2) of Theorem 3.1.

3. Justification of the asymptotic formula (3.1). For the Jacobi matrices $\mathbf{J}\left(0,\left\{\beta_{n}\right\}\right)$ with

$$
\beta_{n} \sim 1 / 2+q n^{-1}, \quad q>0,
$$

the following asymptotics of the function $N_{ \pm}( \pm s ; \mathbf{J})$ is known:

$$
N_{+}(s ; \mathbf{J})=N_{-}(-s ; \mathbf{J}) \sim \frac{q \sqrt{2}}{\sqrt{s-1}}, \quad s \searrow 1
$$


(for the proof, see [15]). Relation (10.2) shows that (10.8) is fulfilled for the matrix $\mathbf{J}_{0}^{\prime}$ with $q=1 / 8$. Therefore, the asymptotic formula (3.1) is a direct consequence of the general formula (10.9).

\section{$\S 11$. Operators $\mathbf{A}_{\alpha}$ For large $\alpha$ : The location of the point spectrum}

Here we prove statement 3) of Theorem 3.2 and statement 2) of Theorem 3.3. These statements describe subsets of the real axis that certainly contain no point spectrum.

First, consider the case where $\alpha \sqrt{2}>d(o)$. Assume that $\Lambda<1 / 2$ is an eigenvalue of the operator $\mathbf{A}_{\alpha}$ and that $W \sim\left\{w_{n}\right\}$ is the corresponding eigenfunction. Then for all $n$ we have $w_{n}=C_{n} \eta_{n}(\Lambda ;$.$) (see (4.17)). By (4.19), \Lambda$ is an eigenvalue if and only if $\left\{C_{n}\right\} \in \ell^{2}$, because otherwise $W \notin \mathfrak{H}$.

The coefficients $C_{n}$ must satisfy (4.18), and $\mu<1$. We denote

$$
\lambda_{+}=-\mu+i \sqrt{1-\mu^{2}}=e^{i \theta}, \quad \frac{\Lambda \mu}{2 \sqrt{1-\mu^{2}}}=\varkappa .
$$

Then, in accordance with (4.25), an arbitrary solution has the following asymptotics:

$$
C_{n}=n^{-1 / 2}\left(P e^{i \theta n} n^{i \varkappa}+Q e^{-i \theta n} n^{-i \varkappa}+o(1)\right), \quad P, Q=\text { const. }
$$

If $|P|^{2}+|Q|^{2} \neq 0$, then the series $\sum_{n}\left|C_{n}\right|^{2}$ diverges for any such sequence. Indeed,

$$
\left|C_{n}\right|^{2}=n^{-1}\left(|P|^{2}+|Q|^{2}+o(1)\right)+2 n^{-1} \operatorname{Re}\left(P \bar{Q} e^{2 i \theta n} n^{2 i \varkappa}\right) .
$$

Here, the first terms on the right give divergent series. At the same time, the sequence of partial sums of the series $\sum_{n} e^{2 i \theta n} n^{2 i \varkappa}$ is bounded (for $\Lambda \neq 0$ the series converges). Thus, if $\left\{C_{n}\right\} \in \ell^{2}$, then $C_{n}=0$ for all $n$. This means that the operator $\mathbf{A}_{\alpha}$ has no eigenvalues $\Lambda<1 / 2$.

For $\alpha \sqrt{2}=d(o)$, i.e., for $\mu=1$, the argument is similar, but now we use statement b) of Lemma 4.4. By (4.26), any solution of (4.18) has asymptotics of the form

$$
C_{n}=n^{-1 / 4}\left(P e^{2 \sqrt{-\Lambda n}}+Q e^{-2 \sqrt{-\Lambda n}}+o(1)\right) .
$$

If $0<\Lambda<1 / 2$, then $\left\{C_{n}\right\} \notin \ell^{2}$, so that $\Lambda$ cannot be an eigenvalue.

For the graph $\Gamma_{d}$ (with central vertex at the point $o$ ), the point spectrum of $\mathbf{A}_{\alpha}$ is empty for any $\alpha \geq d(o) / \sqrt{2}$ (see Proposition 3.4). For a general graph $\Gamma \in \mathfrak{G}$, the operator $\mathbf{A}_{\alpha}$ can have eigenvalues $\Lambda>1 / 2$ for any $\alpha \geq 0$.

Example 11.1. Suppose the graph $\Gamma$ is a circle of length $l=2 \pi \sqrt{2}$, which we view as the interval $[0, l]$ with ends identified. Adding new vertices, we may assume that $\Gamma \in \mathfrak{G}$. Let $o=0$. Then for any $\alpha$ the element $W \sim\{\sin (x / \sqrt{2}), 0,0, \ldots\}$ is an eigenfunction for $\mathbf{A}_{\alpha}$ that corresponds to the eigenvalue $\Lambda=1$. This can be verified by direct calculation. Thus, the eigenvalue is independent of $\alpha$.

This example can be made more interesting by attaching a half-line to our circle at the point $x=\pi \sqrt{2}$. We can extend the function $w_{0}(x)=\sin (x / \sqrt{2})$ to this halfline by zero. Then the new function is still an eigenfunction with the same eigenvalue $\Lambda=1$. This remark shows that the appearance of eigenvalues $\Lambda>1 / 2$ is not related to the compactness of the graph. Rather, this effect is caused by the nontriviality of the fundamental group.

\section{§12. The case of Several oscillators}

This case was the subject of the study in the papers [6, 7, where the simplest "model" example was treated. In that example $\Gamma=\mathbb{R}, K=2$, and the points $o_{1}, o_{2}$ coincide with \pm 1 . However, the approach was of a rather general nature, and it can be expected that 
the results can be extended, with appropriate modifications, to the case where $K$ and the graph $\Gamma \in \mathfrak{G}$ are arbitrary.

In the present section we briefly describe some results obtained in [6, 7]. For simplicity, we assume that the oscillators $h_{1}$ and $h_{2}$ are given by formula (0.1) with $\omega_{1}=\omega_{2}=1$ (this was not assumed in [6, 7]).

The operator $\mathbf{A}_{\alpha_{1}, \alpha_{2}}$ acts in the space $L^{2}\left(\mathbb{R}^{3}\right)$. To analyze the problem, it is natural to use the double series expansion

$$
U\left(x, q_{1}, q_{2}\right)=\sum_{m, n \in \mathbb{N}_{0}} u_{m, n}(x) \chi_{m}\left(q_{1}\right) \chi_{n}\left(q_{2}\right) ;
$$

cf. (2.4). As in $\S 2$, we use the notation $U \sim\left\{u_{m, n}\right\}$. Obviously,

$$
\mathcal{A} U \sim\left\{L_{m, n} u_{m, n}\right\}, \quad\left(L_{m, n} u\right)(x)=-u^{\prime \prime}(x)+(m+n+1) u(x), \quad x \neq \pm 1 .
$$

The conditions at the points \pm 1 reduce to

$$
\begin{aligned}
\sqrt{2}\left[u_{m, n}^{\prime}\right](1) & =\alpha_{1}\left(\sqrt{m+1} u_{m+1, n}(1)+\sqrt{m} u_{m-1, n}(1)\right), \\
\sqrt{2}\left[u_{m, n}^{\prime}\right](-1) & =\alpha_{2}\left(\sqrt{n+1} u_{m, n+1}(-1)+\sqrt{n} u_{m, n-1}(-1)\right) .
\end{aligned}
$$

The selfadjoint realization of the operator $\mathbf{A}_{\alpha_{1}, \alpha_{2}}$ was described in [6]. For any $\alpha_{1}, \alpha_{2} \geq 0$, let $\mathcal{D}_{\alpha_{1}, \alpha_{2}}$ denote the set of elements $U \sim\left\{u_{m, n}\right\}$ such that $u_{m, n} \in H^{1}(\mathbb{R})$ for all $m, n \in \mathbb{N}_{0}$, the restriction of $u_{m, n}$ to the intervals $(-\infty,-1),(-1,1)$, and $(1, \infty)$ belongs to $H^{2}$,

$$
\sum_{m, n} \int_{\mathbb{R}}\left|L_{m, n} u_{m, n}\right|^{2} d x<\infty
$$

and, finally, conditions (12.2) and (12.3) are fulfilled.

The following result is an analog of Theorem 2.1.

Theorem 12.1. Let $\mathcal{A}$ be the operator defined by (12.1). For any $\alpha_{1}, \alpha_{2} \geq 0$, the operator $\mathbf{A}_{\alpha_{1}, \alpha_{2}}:=\mathcal{A} \uparrow \mathcal{D}_{\alpha_{1}, \alpha_{2}}$ is selfadjoint in $L^{2}\left(\mathbb{R}^{3}\right)$.

For the proof, first we repeat the argument used in $\S 5$ to prove Theorem 2.1. As a result, we obtain a system of linear homogeneous algebraic equations. This is a recurrence system with respect to the two parameters $m, n$. We need to show that this system has no nontrivial solutions in $\ell^{2}$. The structure of the system does not allow us to apply the Birkhoff-Adams theorem (as was done in $§ 5$ ). However, this system can be regarded as a weak perturbation of a certain splitting system. The analysis of the latter reduces to an application of the already known result for the case of one oscillator. This leads to the required statement.

The analysis of the spectrum of $\mathbf{A}_{\alpha_{1}, \alpha_{2}}$ is based on two simple observations. First, if one of the parameters $\alpha_{1}, \alpha_{2}$ is zero, then the operator splits into the orthogonal sum of operators corresponding to the case of one oscillator. For example,

$$
\mathbf{A}_{\alpha, 0}=\sum_{n \in \mathbb{N}_{0}}^{\oplus}\left(\mathbf{A}_{\alpha}+(n+1 / 2)\right),
$$

where $\mathbf{A}_{\alpha}$ is the operator (2.1), (2.2) for $(\Gamma, o)=(\mathbb{R}, 1)$. Using the decomposition (12.4) and the results of $\S 3$, we obtain a complete description of the point spectrum and the absolutely continuous spectrum for the operators $\mathbf{A}_{\alpha, 0}$ and $\mathbf{A}_{0, \alpha}$. In particular, Proposition 3.4 shows that for $\alpha<\sqrt{2}$ only the first term in (12.4) contributes to the point spectrum of $\mathbf{A}_{\alpha, 0}$ :

$$
N_{-}\left(1 ; \mathbf{A}_{\alpha, 0}\right)=N_{-}\left(1 / 2 ; \mathbf{A}_{\alpha}\right), \quad \alpha<\sqrt{2} .
$$

Second, the interaction between two oscillators attached to the graph at different points is very weak. Therefore, we can expect that information about the spectrum of 
the initial operator $\mathbf{A}_{\alpha_{1}, \alpha_{2}}$ can be deduced from the knowledge of the spectra of the operators $\mathbf{A}_{\alpha_{1}, 0}$ and $\mathbf{A}_{0, \alpha_{2}}$. For the most part, these expectations come true, but the realization of this scheme is different for the absolutely continuous spectrum and the point spectrum.

The absolutely continuous spectrum of $\mathbf{A}_{\alpha_{1}, \alpha_{2}}$ was investigated in [6]. It was found that this spectrum is the union of $\sigma_{\text {a.c. }}\left(\mathbf{A}_{\alpha_{1}, 0}^{+}\right)$and $\sigma_{\text {a.c. }}\left(\mathbf{A}_{0, \alpha_{2}}^{-}\right)$, where $\mathbf{A}_{\alpha_{1}, 0}^{+}$and $\mathbf{A}_{0, \alpha_{2}}^{-}$ are the operators defined by expansions similar to (12.4) but for the two "halves" of the graph $\Gamma$ rather then for the entire graph.

More precisely, let $\mathbf{A}_{\alpha}^{ \pm}$be the operator of the form (2.1)-(2.2) for the configuration $(\Gamma, o)=\left(\mathbb{R}_{ \pm}, \pm 1\right)$ with the additional Dirichlet condition $U(0, q)=0$. We put

$$
\widehat{\mathbf{A}}_{\alpha}^{ \pm}=\sum_{n \in \mathbb{N}_{0}}^{\oplus}\left(\mathbf{A}_{\alpha}^{ \pm}+(n+1 / 2)\right), \quad \widehat{\mathbf{A}}_{\alpha_{1}, \alpha_{2}}:=\widehat{\mathbf{A}}_{\alpha_{1}}^{+} \oplus \widehat{\mathbf{A}}_{\alpha_{2}}^{-} .
$$

In [6] it was proved that for any $\Lambda \neq \bar{\Lambda}$ we have

$$
\left(\widehat{\mathbf{A}}_{\alpha_{1}, \alpha_{2}}-\Lambda\right)^{-3}-\left(\mathbf{A}_{\alpha_{1}, \alpha_{2}}-\Lambda\right)^{-3} \in \mathfrak{S}_{1} \text {. }
$$

In accordance with a well-known result by Kato (see, e.g., Theorem 6.5.1 and Remark 6.5.2 in the book 16]), relation (12.5) implies the existence of complete isometric wave operators for the pair $\widehat{\mathbf{A}}_{\alpha_{1}, \alpha_{2}}, \mathbf{A}_{\alpha_{1}, \alpha_{2}}$. Consequently, the absolutely continuous spectra and the corresponding multiplicity functions for both operators coincide. This leads to a complete description of the absolutely continuous spectrum of the operator $\mathbf{A}_{\alpha_{1}, \alpha_{2}}$. In particular, if $\max \left(\alpha_{1}, \alpha_{2}\right)>\sqrt{2}$, then $\sigma_{\text {a.c. }}\left(\mathbf{A}_{\alpha_{1}, \alpha_{2}}\right)=\mathbb{R}$ and $\mathfrak{m}_{\text {a.c. }}\left(\lambda ; \mathbf{A}_{\alpha_{1}, \alpha_{2}}\right) \equiv \infty$. In all other cases, the multiplicity function $\mathfrak{m}_{\text {a.c. }}\left(\lambda ; \mathbf{A}_{\alpha_{1}, \alpha_{2}}\right)$ is finite for all $\lambda$. Moreover, $\sigma_{\text {a.c. }}\left(\mathbf{A}_{\alpha_{1}, \alpha_{2}}\right)=\left[\lambda_{0}, \infty\right)$, where $\lambda_{0}=1$ for $\alpha_{1}, \alpha_{2}<\sqrt{2}, \lambda_{0}=1 / 2$ for $\alpha_{1}<\alpha_{2}=\sqrt{2}$ and for $\alpha_{2}<\alpha_{1}=\sqrt{2}$, and $\lambda_{0}=0$ for $\alpha_{1}=\alpha_{2}=\sqrt{2}$. It is not difficult to write an explicit expression for the multiplicity function, but we omit this.

The point spectrum of $\mathbf{A}_{\alpha_{1}, \alpha_{2}}$ was investigated in [7] for the case where $0 \leq \alpha_{1}, \alpha_{2} \leq$ $\sqrt{2}$. For such values of the parameters, the variational approach applies. As in the case of $K=1$ (see $\S 9$ ), it is convenient to write the quadratic form $\mathbf{a}_{\alpha_{1}, \alpha_{2}}$ of the operator $\mathbf{A}_{\alpha_{1}, \alpha_{2}}$ (with $\omega_{1}=\omega_{2}=1$ ) as follows:

$$
\mathbf{a}_{\alpha_{1}, \alpha_{2}}[U]=\mathbf{a}_{0,0}[U]+\alpha_{1} \mathbf{b}_{1}[U]+\alpha_{2} \mathbf{b}_{2}[U]
$$

where

$$
\mathbf{a}_{0,0}[U]=\sum_{m, n \in \mathbb{N}_{0}} \int_{\mathbb{R}}\left(\left|u_{m, n}^{\prime}\right|^{2}+(m+n+1)\left|u_{m, n}\right|^{2}\right) d x,
$$

and the quadratic forms $\mathbf{b}_{1}[U]$ and $\mathbf{b}_{2}[U]$ are expressed by sums similar to the sum (9.1).

The quadratic form $\mathbf{a}_{0,0}$ is positive definite and closed on the natural domain Dom $\mathbf{a}_{0,0}$, which is defined by the requirement that the sum on the right in (12.6) be finite. The following statement is an analog of Lemma 9.4 .

Theorem 12.2. Let $\alpha_{1}, \alpha_{2} \leq \sqrt{2}$. Then the quadratic form $\alpha_{1} \mathbf{b}_{1}+\alpha_{2} \mathbf{b}_{2}$ is bounded relative to the form $\mathbf{a}_{0,0}$ with form-bound not exceeding one. More precisely, there exists a constant $R \geq 0$ independent of $\alpha_{1}, \alpha_{2}$ and such that

$$
\left|\alpha_{1} \mathbf{b}_{1}[U]+\alpha_{2} \mathbf{b}_{2}[U]\right| \leq \mathbf{a}_{0,0}[U]+R\|U\|_{L^{2}\left(\mathbb{R}^{3}\right)}^{2} \quad \text { for all } U \in \operatorname{Dom} \mathbf{a}_{0,0} .
$$

Consequently, for such $\alpha_{1}, \alpha_{2}$ the quadratic form $\mathbf{a}_{\alpha_{1}, \alpha_{2}}[U]$ is lower semibounded and closed on Dom $\mathbf{a}_{0,0}$.

Comparing this theorem and Lemma 9.4, we see that the leading term on the right in estimate (12.7) is the same as in the case where $K=1$. The reason is that, to estimate the forms $\mathbf{b}_{1}$ and $\mathbf{b}_{2}$, it suffices to use integrals over arbitrarily small neighborhoods 
of the points \pm 1 , respectively. This is one more manifestation of the locality property discussed in Subsection 4.4.

By Theorem 12.2, for $\alpha_{1}, \alpha_{2} \leq \sqrt{2}$ the operator $\mathbf{A}_{\alpha_{1}, \alpha_{2}}$ can be defined via its quadratic form $\mathbf{a}_{\alpha_{1}, \alpha_{2}}$. Also, this approach makes it possible to obtain information about the spectrum in the same way as was done in $\S \S 9,10$ in the case of $K=1$. In particular, it can be shown that for $\alpha_{1}, \alpha_{2}<\sqrt{2}$ the spectrum below the threshold point $\lambda_{0}=1$ is nonempty and consists of finitely many eigenvalues of finite multiplicity. In particular, for such $\alpha_{1}, \alpha_{2}$ the essential spectrum and the absolutely continuous spectrum coincide geometrically.

It is possible to give a complete analysis of the behavior of the function $N_{-}\left(1 ; \mathbf{A}_{\alpha_{1}, \alpha_{2}}\right)$ as $\alpha_{1}, \alpha_{2}$ tend to $\sqrt{2}$ independently. By using the fact that the oscillators interact weakly, we can obtain the asymptotics of the function $N_{-}\left(1 ; \mathbf{A}_{\alpha_{1}, \alpha_{2}}\right)$ with respect to both parameters $\alpha_{1} \rightarrow \sqrt{2}, \alpha_{2} \rightarrow \sqrt{2}$. This asymptotic formula is uniform in the domain $0<\alpha_{1}<\varphi\left(\alpha_{2}\right), 0<\alpha_{2}<\varphi\left(\alpha_{1}\right)$, where $\varphi$ is an increasing function on $(0, \sqrt{2}]$ such that $\varphi(\sqrt{2})=\sqrt{2}$ and $\varphi^{(l)}(\sqrt{2})=0$ for all $l$. For precise formulations, see [7].

\section{§13. Proof of Lemmas 4.1 and 4.2}

We start with a simple remark. Let $\Gamma \in \mathfrak{G}$ be an arbitrary graph, and let $o \in \Gamma$ be its marked vertex. Obviously, on every edge $e \subset \mathcal{S}(o)$ the function $u_{e}=\left.g(\zeta ; ., o)\right|_{e}$ can be written as

$$
u_{e}(t)=e^{-\sqrt{\zeta} t}-C_{\zeta, e} \sinh (\sqrt{\zeta} t),
$$

where $C_{\zeta, e}$ is a constant. Then

$$
r(\zeta ; o)=\sqrt{\zeta}\left(d(o)+\sum_{e \subset \mathcal{S}(o)} C_{\zeta, e}\right) .
$$

Proof of Lemma 4.1. For $\zeta=k^{2}>0$ we can use the variational description of the Green function. Namely, the function $g_{k}(x):=g\left(k^{2} ; x, o\right)$ is the only function of class $H^{1}(\Gamma)$ that minimizes the quadratic functional

$$
a_{k}[g]:=\int_{\Gamma}\left(\left|g^{\prime}(x)\right|^{2}+k^{2}|g(x)|^{2}\right) d x
$$

under the condition $g(o)=1$. Then the standard calculations show that

$$
0<g\left(k^{2} ; x, o\right)<1 \quad \text { for all } x \in \Gamma, x \neq o .
$$

In its turn, (13.3) implies that for $\zeta=k^{2}>0$ we have

$$
0 \leq C_{k^{2}, e} \leq C_{k^{2}, e}^{0}:=\frac{e^{-k|e|}}{\sinh (k|e|)}
$$

on every edge $e \subset \mathcal{S}(o)$ of finite length. Indeed, for $C_{k^{2}, e}<0$ the left inequality in (13.3) would fail on the entire edge $e$, and for $C_{k^{2}, e}>C_{k^{2}, e}^{0}$ the right inequality in (13.3) would fail at the vertex opposite to the vertex $o$. The changes arising if $|e|=\infty$ are obvious, and we do not discuss them. Estimate (4.8) is a direct consequence of (13.2) and (13.4).

Relations (13.4) imply the following useful inequality for the function $u_{e}=\left.g\left(k^{2} ; ., o\right)\right|_{e}$ :

$$
\frac{\sinh (k(|e|-t))}{\sinh (k|e|)} \leq u_{e}(t) \leq e^{-k t}, \quad 0 \leq t \leq|e| .
$$

Now we define a function $g^{*}$ on the graph $\Gamma$. Namely, we let $g^{*}$ be equal to the function occurring on the left-hand side of (13.5) on every edge $e \subset \mathcal{S}(o)$, and off $\mathcal{S}(o)$ we put 
$g^{*}(x)=0$. Then $g^{*} \in H^{1}(\Gamma), g^{*}(o)=1$, and

$$
a_{k}\left[g^{*}\right]=k \sum_{e \subset \mathcal{S}(o)} \operatorname{coth}(k|e|)
$$

The extremal property of the function $g\left(k^{2} ; ., o\right)$ implies that $a_{k}\left[g\left(k^{2} ; ., o\right)\right] \leq a_{k}\left[g^{*}\right]$. Since $k^{2}\left\|g\left(k^{2} ; ., o\right)\right\|_{L^{2}(\Gamma)}^{2} \leq a_{k}\left[g_{k}\right]$, this leads to the upper bound in (4.9). To obtain the lower bound, it suffices to estimate the integral over $\mathcal{S}(o)$ from below with the help of the left inequality in (13.5).

It looks unusual that, in the above argument, the left inequality in (13.5) was employed to obtain both the lower and the upper bound in (4.9).

We pass to the case of nonreal $\zeta$. First, we consider the nonhomogeneous boundary problem

$$
-w^{\prime \prime}(x)+\zeta w(x)=f(x), \quad x \notin \mathcal{V},
$$

with the conditions

$$
\left[w^{\prime}\right](x)=0, \quad x \in \mathcal{V}, \quad x \neq o ; \quad w(o)=0 .
$$

As in $\S 4$, we put $k=\operatorname{Re} \sqrt{\zeta} k>0$. Also, let $\beta=\operatorname{Im} \sqrt{\zeta}$. Using (13.6), after integration by parts we obtain

$$
\int_{\Gamma}\left(\left|w^{\prime \prime}\right|^{2}+2\left(k^{2}-\beta^{2}\right)\left|w^{\prime}\right|^{2}+\left(k^{2}+\beta^{2}\right)^{2}|w|^{2}\right) d x=\int_{\Gamma}|f|^{2} d x .
$$

For $|\beta| \leq k$, identity (13.8) implies the following inequality for the solution $w$ :

$$
\int_{\Gamma}|w|^{2} d x \leq k^{-4} \int_{\Gamma}|f|^{2} d x
$$

Proof of Lemma 4.2. For notational simplicity, we assume that the conditions of the lemma are fulfilled with $c=1$. The function

$$
w=g(\zeta ; x, o)-g\left(k^{2} ; x, o\right)
$$

solves problem (13.6), (13.7) with $f(x)=-\left(2 i k \beta-\beta^{2}\right) g\left(k^{2} ; x, o\right)$. If $|\operatorname{Im} \zeta| \leq 1$, then $|\beta| \leq(2 k)^{-1}$. Therefore, by (4.9) and (13.9),

$$
\int_{\Gamma}|w|^{2} d x \leq k^{-4}\left(4 k^{2} \beta^{2}+\beta^{4}\right) \int_{\Gamma}\left|g\left(k^{2} ; x, o\right)\right|^{2} d x \leq 2 M_{2} d(o) k^{-5} .
$$

This leads to (4.11).

The proof of (4.10) is more cumbersome. We fix one of the edges $e \subset \mathcal{S}(o)$, identifying it with the interval $[0, l]$. Omitting the index $e$ and using the representation (13.1), we write

$$
w(t)=e^{-k t}\left(e^{-i \beta t}-1\right)-\left(C_{\zeta}-C_{k^{2}}\right) \sinh (k t+i \beta t)-C_{k^{2}}(\sinh (k t+i \beta t)-\sinh (k t)) .
$$

The elementary estimates

$$
|\sinh (\nu+i \tau)| \geq|\sinh \nu| ; \quad|\sinh (\nu+i \tau)-\sinh \nu| \leq 2 \cosh \nu,
$$

and inequality (13.4) imply that

$$
\begin{aligned}
|w(t)| & \geq\left|C_{\zeta}-C_{k^{2}}\right| \sinh (k t)-2 \frac{e^{-k l}}{\sinh (k l)} \cosh (k t)-2 e^{-k t} \\
& =\left|C_{\zeta}-C_{k^{2}}\right| \sinh (k t)-2 \frac{\cosh (k(l-t))}{\sinh (k l)} .
\end{aligned}
$$


Since the inequality $A \geq B-C$ for any real $A, B, C$ yields $2 A^{2} \geq B^{2}-2 C^{2}$, we get

$$
2|w(t)|^{2} \geq\left|C_{\zeta}-C_{k^{2}}\right|^{2} \sinh ^{2}(k t)-8 \frac{\cosh ^{2}(k(l-t))}{\sinh ^{2}(k l)} .
$$

Now, we integrate this inequality over every edge $e \subset \mathcal{S}(o)$ and sum the results. Using (13.10), we obtain the inequality

$$
\frac{4 M_{2} d(o)}{k^{5}} \geq \sum_{e \subset \mathcal{S}(o)}\left(\left|C_{\zeta}-C_{k^{2}}\right|^{2}\left(\frac{\sinh (2 k|e|)}{2 k}-|e|\right)-\frac{8}{\sinh ^{2}(k|e|)}\left(\frac{\sinh (2 k|e|)}{2 k}+|e|\right)\right) .
$$

The coefficient of $\left|C_{\zeta}-C_{k^{2}}\right|^{2}$ grows exponentially as $k$ increases. At the same time, the "free term" on the right-hand side stays bounded. Therefore,

$$
\sum_{e \subset \mathcal{S}(o)}\left|C_{\zeta}-C_{k^{2}}\right|^{2}=O\left(k^{-4} e^{-2 k \varepsilon(\Gamma, o)}\right), \quad k \rightarrow \infty .
$$

By (13.2),

$$
\left|r(\zeta ; o)-\sqrt{\zeta}\left(d(o)+\sum_{e \subset \mathcal{S}(o)} C_{k^{2}, e}\right)\right| \leq|\zeta|^{1 / 2} \sum_{e \subset \mathcal{S}(o)}\left|C_{\zeta}-C_{k^{2}}\right| .
$$

Now, relation (4.10) follows from (4.8) and (13.11).

\section{REFERENCES}

[1] M. Sh. Birman and M. Z. Solomyak, Spectral theory of selfadjoint operators in Hilbert space, Leningrad. Univ., Leningrad, 1980; English transl., Reidel, Dordrecht, 1987. MR0609148 (82k:47001) MR:1192782(93g:47001)

[2] M. Sh. Birman and S. B. Ėntina, Stationary approach in abstract scattering theory, Izv. Akad. Nauk SSSR Ser. Mat. 31 (1967), no. 2, 401-430; English transl., Math. USSR-Izv. 1 (1967), no. 1, 391-420. MR0209895 (35:790)

[3] D. J. Gilbert and D. B. Pearson, On subordinacy and analysis of the spectrum of one-dimensional Schrödinger operators, J. Math. Anal. Appl. 128 (1987), no. 1, 30-56. MR0915965 (89a:34033)

[4] I. Ts. Gokhberg and M. G. Krein, Introduction to the theory of linear nonselfadjoint operators, "Nauka," Moscow, 1965; English transl., Transl. Math. Monogr., vol. 18, Amer. Math. Soc., Providence, RI, 1969. MR0220070 (36:3137) MR0246142 (39:7447)

[5] S. N. Elaydi, An introduction to difference equations, Springer-Verlag, New York, 1999. MR1711587 (2001g:39001)

[6] W. D. Evans and M. Solomyak, Smilansky's model of irreversible quantum graphs. I. The absolutely continuous spectrum, J. Phys. A 38 (2005), 4611-4627. MR2147079

[7] _ Smilansky's model of irreversible quantum graphs. II. The point spectrum, J. Phys. A 38 (2005), 7661-7675. MR2169482

[8] S. Khan and D. B. Pearson, Subordinacy and spectral theory for infinite matrices, Helv. Phys. Acta 65 (1992), no. 4, 505-527. MR 1179528 (94a:47066)

[9] P. Kuchment, Graph models for waves in thin structures, Waves Random Media 12 (2002), no. 4, R1-R24. MR1937279 (2003h:35215)

[10] S. N. Naboko, Conditions for the existence of wave operators in the nonselfadjoint case, Wave Propagation. Scattering Theory, Probl. Mat. Fiz., vyp. 12, Leningrad. Univ., Leningrad, 1987, pp. 132-155; English transl., Amer. Math. Soc. Transl. (2), vol. 157, Amer. Math. Soc., Providence, RI, 1993, pp. 127-149. MR0923975 (90b:47019)

[11] _ Uniqueness theorems for operator-valued functions with positive imaginary part, and the singular spectrum in the selfadjoint Friedrichs model, Ark. Mat. 25 (1987), 115-140. MR0918381 (89e:47028)

[12] S. N. Naboko and M. Solomyak, On the absolutely continuous spectrum of a family of operators appearing in the theory of irreversible quantum systems, Proc. London Math. Soc. (3) 92 (2006), $251-272$.

[13] U. Smilansky, Irreversible quantum graphs, Waves Random Media 14 (2004), S143-S153. MR 2042550 (2004m:82087)

[14] M. Solomyak, On a differential operator appearing in the theory of irreversible quantum graphs, Waves Random Media 14 (2004), S173-S185. MR2042551(2005a:35213) 
[15] - The discrete spectrum of a family of differential operators, Funktsional. Anal. i Prilozhen. 38 (2004), no. 3, 70-78; English transl., Funct. Anal. Appl. 38 (2004), no. 3, 217-223. MR2095135 (2005h:47088)

[16] D. R. Yafaev, Mathematical scattering theory. General theory, S.-Peterburg. Univ., St. Petersburg, 1994; English transl., Transl. Math. Monogr., vol. 105, Amer. Math. Soc., Providence, RI, 1992. MR1784870(2001e:47015) MR1180965 (94f:47012).

Department of Mathematics, The Weizmann Institute of Science, Rehovot 76100, Israel

E-mail address: michail.solomyak@weizmann.ac.il

Received 21/DEC/2004

Translated by R. SHTERENBERG 